\title{
Salmonella enterica serovar Choleraesuis vector delivering SaoA antigen confers protection against Streptococcus suis serotypes 2 and 7 in mice and pigs
}

\author{
Yu-an $\mathrm{Li}^{1,2}$, Zhenying $\mathrm{Ji}^{1,2}$, Xiaobo Wang ${ }^{1,2}$, Shifeng Wang ${ }^{3}$ and Huoying Shi ${ }^{1,2^{*}}$
}

\begin{abstract}
Streptococcus suis is one of the major pathogens that cause economic losses in the swine industry worldwide. However, current bacterins only provide limited prophylactic protection in the field. An ideal vaccine against $S$. suis should protect pigs against the clinical diseases caused by multiple serotypes, or at least protect against the dominant serotype in a given geographic region. A new recombinant Salmonella enterica serotype Choleraesuis vaccine vector, rSC0011, that is based on the regulated delayed attenuation system and regulated delayed antigen synthesis system, was developed recently. In this study, an improved recombinant attenuated Salmonella Choleraesuis vector, rSC0016, was developed by incorporating a sopB mutation to ensure adequate safety and maximal immunogenicity. In the spleens of mice, rSC0016 colonized less than rSC0011. rSC0016 and rSC0011 colonized similarly in Peyer's patches of mice. The recombinant vaccine rSC0016(pS-SaoA) induced stronger cellular, humoral, and mucosal immune responses in mice and swine against SaoA, a conserved surface protein that is present in many S. suis serotypes, than did rSC0011 (pS-SaoA) without SopB or rSC0018(pS-SaoA), which is an avirulent, chemically attenuated vaccine strain. rSC0016(pS-SaoA) provided 100\% protection against S. suis serotype 2 in mice and pigs, and full cross-protection against SS7 in pigs. This new vaccine vector provides a foundation for the development of a universal vaccine against multiple serotypes of $S$. suis in pigs.
\end{abstract}

\section{Introduction}

Streptococcus suis (S. suis) strains are classified into 35 serotypes based on the capsular polysaccharide antigens. Globally, the predominant S. suis serotype isolated from clinical cases of the disease in pigs is serotype 2, followed by serotypes $9,3,1 / 2$, and 7 , together with $15.5 \%$ nontypable strains [1]. To date, only vaccination with $S$. suis bacterins is performed to prevent $S$. suis infection in swine. Although several studies have demonstrated homologous protection [2], the procedure usually has a high rate of failure because the antigenicity of the vaccine is damaged by heat and formalin processing, which leads

\footnotetext{
*Correspondence: hyshi@yzu.edu.cn

${ }^{1}$ College of Veterinary Medicine, Yangzhou University, Yangzhou 225009, Jiangsu, China

Full list of author information is available at the end of the article
}

to the production of antibodies that are not associated with protection and/or are cross-reactive $[3,4]$. Current vaccine research for $S$. suis focuses on subunit vaccine based on conserved proteins among different serotypes to protect pigs against clinical diseases caused by various S. suis serotypes, or protect against the dominant serotype in a given geographic region. However, the cost of many vaccines made from conserved proteins in veterinary medicine and swine medicine is relatively high compared to traditional bacterins.

To date, at least 24 subunit vaccine candidates have been identified [5-22]. Most of them were tested in mouse models $[5,8,11,12,15,17-22]$, and only a few were evaluated in pigs $[5,7,9,10,12,14,16,19,23,24]$. The results showed that the protection conferred in swine is usually lower than that conferred in mice [10, 23]. Therefore, it is necessary to evaluate the candidate 
antigen in the target animal, pig. Moreover, the capacities to induce cross-protection were evaluated for a few antigens. Of these antigens, the surface-anchored protein (Sao) is a membrane-anchored bacterial protein reacting with 28 of 33 S. suis serotypes and 25 of 26 serotype 2 isolates, suggesting that it is a highly conserved protein in $S$. suis [16]. The effectiveness of Sao as a vaccine candidate depends upon the adjuvant used. Sao formulated with Emulsigen-Plus ${ }^{\circledR}$ provided no protection against S. suis serotype 2 (SS2) because the induced antibodies lacked opsonizing activity against SS2 [16], whereas Sao formulated with the Quil-A adjuvant partially protected pigs against aerosol challenge with SS2 because it induced opsonizing antibodies [24]. So far, Sao is the only protein that has been shown to induce cross-protection against serotype 1 and 7 strains in mice and pigs [19]. These results indicate that the efficacy of the protection induced by Sao as a subunit vaccine candidate may depend on the choice of appropriate vector or adjuvant.

Streptococcus suis is an encapsulated microorganism. Host protection against infection by $S$. suis is primarily mediated by opsonophagocytosis, which is mainly associated with a Th1-type immune response [25]. Salmonella has excellent adjuvant attributes and induces high mucosal, cellular and humoral responses [26]. However, live attenuated Salmonella, traditionally generated by deletion of its virulence genes, can lack a balance between the loss of its disease-causing ability and the ability to persist and induce immune responses [27], and is therefore not an ideal vaccine vector. In addition, constitutive high-level antigen synthesis causes a metabolic burden to the vaccine vector strain that can reduce the vaccine strain's ability to interact with host lymphoid tissues, resulting in a compromised immune response [28]. To solve these problems, the regulated delayed attenuation system was developed to make a strain displaying features of wild-type virulent strains of Salmonella at the time of immunization to enable strains to effectively colonize lymphoid tissues and then exhibit a regulated delayed attenuation in vivo to preclude inducing disease symptoms [29]. The regulated delayed antigen synthesis system could regulate antigen gene expression and permit high levels of antigen synthesis only after the vaccine strain reaches its target tissues [30]. The regulated delayed attenuation strategy includes a smooth-to-rough phenotypic change in lipopolysaccharides (LPS) with the presence or absence of mannose [31], and the replacement of the promoters of some virulence genes (fur, crp, and phoPQ) with a tightly regulated $\operatorname{araC} \mathrm{P}_{\mathrm{BAD}}$ cassette so that the expression of these genes is dependent on arabinose supplement during in vitro growth [29]. Following the colonization of the lymphoid tissues, these virulence-associated proteins cease to be synthesized because no mannose or arabinose is present in vivo [32]. Therefore, attenuation manifests gradually in vivo, precluding the induction of disease symptoms and inducing the desired antigen-specific immune responses [33]. Furthermore, an araC $\mathrm{P}_{\mathrm{BAD}}$ cassette was used to regulate the expression of chromosomal lacI repressor gene, which binds to $\mathrm{P}_{\text {trc }}$ on an expression plasmid and blocks antigen synthesis in the presence of arabinose in vitro. Once Salmonella reaches arabinose-free, host immunocompetent sites, the concentration of LacI decreases with each cell division, allowing upregulation of antigen synthesis and induction of desired antigen-specific immune responses [30].

In a previous study, a recombinant attenuated Salmonella enterica serotype Choleraesuis (Salmonella Choleraesuis) vaccine strain $\mathrm{rSC} 0011$ with the regulated delayed strategy in the wild-type Salmonella Choleraesuis C78-3 background was constructed [34]. Strain rSC0011, carrying 6-phosphogluconate dehydrogenase (6-PGD) gene of SS2, conferred $100 \%$ protection against an SS2 challenge in mice [34], but less than $50 \%$ protection in pigs (unpublished data). To increase the efficacy of our vaccine strain, a sopB mutation was introduced into rSC0011 vector to generate a new recombinant attenuated Salmonella Choleraesuis strain rSC0016 based on the previous report that the $\operatorname{sop} B$ mutation could improve the immunogenicity of Salmonella vaccine vector in mice [35]. The heterologous antigen gene, saoA from S. suis, was cloned into the $\mathrm{Asd}^{+}$expression plasmid pYA3493, generating plasmid pS-SaoA, which was used to transform the vector strain $\mathrm{rSC} 0016$, generating the candidate vaccine rSC0016 (pS-SaoA). The virulence and immune attributes of rSC0016 (pS-SaoA) in mice and piglets, and its crossprotection against SS2 and SS7 in piglets were evaluated. Our results showed that the improved recombinant attenuated Salmonella Choleraesuis, which combines the regulated delayed strategy and the $\operatorname{sop} B$ deletion, is more immunogenic than the parent strain rSC0011. It induces cross-protection against multiple serotypes of $S$. suis when delivering the heterologous protective antigen Sao. This could be a candidate for a universal vaccine against S. suis.

\section{Materials and methods}

\section{Ethics statement}

All animal experiments were approved by the Jiangsu Administrative Committee for Laboratory Animals (permission number SYXK-SU-2007-0005) and complied with the Jiangsu Laboratory Animal Welfare and Ethics guidelines of the Jiangsu Administrative Committee of Laboratory Animals. 
Strains, plasmids and culture conditions

The strains and plasmids used in this study are described in Table 1. E. coli strain X7213, plasmid pRE112, and pYA3493 were provided by Dr Roy Curtiss $3^{\text {rd }} S$. suis serotype 2 (SS2, CVCC3928) and S. suis serotype 7 (SS7) were purchased from China Institute of Veterinary Drug Control (Beijing, China). Strain C500, a governmentapproved live attenuated Salmonella Choleraesuis vaccine strain, was used as an attenuation control [36, 37]. Plasmid pYA3493 is an $\mathrm{Asd}^{+}$vector, and plasmid pSSaoA, derived from pYA3493, carries a $s a o A$ gene from SS2. LB medium [38], MacConkey agar (Difco), Nutrient broth (NB) and agar (Difco), and minimal salts medium and agar [39] were used for routine phenotype characterization. Nutrient broth (NB) and agar (Difco) are devoid of arabinose and mannose. When required, media were supplemented with chloramphenicol $(\mathrm{Cm} ; 25 \mu \mathrm{g} / \mathrm{mL})$, kanamycin (Kan; $50 \mu \mathrm{g} / \mathrm{mL}$ ), 2,6-diaminopimelic acid (DAP; $50 \mu \mathrm{g} / \mathrm{mL})$, L-arabinose $(0.2 \% \mathrm{wt} / \mathrm{vol})$, D-mannose $(0.2 \% \mathrm{wt} / \mathrm{vol}), \mathrm{D}$-lactose $(1 \% \mathrm{wt} / \mathrm{vol}), \mathrm{D}$-maltose $(1 \% \mathrm{wt} /$ $\mathrm{vol})$, or sucrose $(5 \% \mathrm{wt} / \mathrm{vol})$. Selenite broth or Tetrathionate broth (Difco), with or without supplements, was used for enrichment of Salmonella Choleraesuis from animal tissues. Strains were grown and prepared as previously described [34]. Briefly, Salmonella Choleraesuis vaccine vector strains harboring plasmid $\mathrm{pS}-\mathrm{SaoA}$ (expression vector) or pYA3493 (empty vector) were grown in LB broth with $0.2 \%$ arabinose and $0.2 \%$ mannose. SS2 or SS7 were cultured on brain heart infusion (BHI) agar containing $5 \%$ sheep blood or in Todd-Hewitt broth plus 0.5\% yeast extract (Oxoid, Nepean, Ontario, Canada) [40].

\section{Construction and characterization of Salmonella Choleraesuis mutant strains}

Five mutations were introduced into wild-type Salmonella Choleraesuis C78-3 by conjugation with $E$. coli X7213 harboring different suicide vectors as previously described [29, 33, 34]. Plasmid constructs and primers for $\Delta p m i, \Delta \mathrm{P}_{\text {crp }}:$ TT araC $\mathrm{P}_{\mathrm{BAD}}$ crp, $\Delta$ rela::araC $\mathrm{P}_{\mathrm{BAD}}$ lacI TT, $\Delta \operatorname{sop} B$, and $\triangle a s d A$ have been described $[34,41]$. The upstream primers of $\operatorname{sop} B$ mutation in Salmonella Choleraesuis (P1: 5'-CGCAGAGCTCATATCACCTATAATTATC-3'; P2: 5'- ATTAGGTACCAGCAGTATTGTCTGCGTCAGC $-3^{\prime}$ ) (Figure 1A) have not been previously described; the upstream sequences are different between Salmonella Choleraesuis and Salmonella Typhimurium.

To detect the effect of $\operatorname{sop} B$ deletion, the fluid secretion in rabbit ileal loops after inoculation with wildtype strain C78-3, rSC0017 (Salmonella Choleraesuis C78-3 with $\operatorname{sop} B$ mutation), vaccine strains $\mathrm{rSC} 0018$ (pSSaoA), rSC0011(pS-SaoA), and rSC0016(pS-SaoA) were

Table 1 Strains and plasmids

\begin{tabular}{|c|c|c|}
\hline Strain or plasmid & Relevant characteristics or genotype & Source or references \\
\hline \multicolumn{3}{|l|}{ E. coli strains } \\
\hline BL21 & $\mathrm{F}^{-}$pThsdSB(rB- $\mathrm{mB}^{-}$aldcm (DE3) & Invitrogen \\
\hline$x 7213$ & thi-1 thr-1 leuB6 fhuA21 lacY1 gInV44 asdA4 recA1 RP4 2-Tc::Mu pir; Km ${ }^{r}$ & Dr Curtiss gift \\
\hline \multicolumn{3}{|l|}{ Salmonella Choleraesuis } \\
\hline C78-3 & Wild type, virulent, CVCC79103 & {$[65]$} \\
\hline C500 & Salmonella Choleraesuis vaccine strain attenuated by chemical mutation, CVCC79500 & {$[37]$} \\
\hline rSC0010 & $\Delta \mathrm{P}_{\mathrm{Cr} 5527}:: \mathrm{TT}$ araC $\mathrm{P}_{\mathrm{BAD}} \operatorname{crp} \Delta p m i-2426 \Delta$ relA199::araC $\mathrm{P}_{\mathrm{BAD}}$ lacl TT & {$[34]$} \\
\hline rSC0011 & $\Delta \mathrm{P}_{\mathrm{crp} 527 .:}: \mathrm{TT}$ araC $\mathrm{P}_{\mathrm{BAD}} \operatorname{crp} \Delta p m i-2426 \Delta$ relA199::araC $\mathrm{P}_{\mathrm{BAD}}$ lacl TT $\triangle$ asdA33 & {$[34]$} \\
\hline rSC0013 & rSC0010 $\triangle$ sopB1686 & This work \\
\hline rSC0016 & rSC0013 $\triangle a s d A 33$ & This work \\
\hline rSC0017 & C78-3 $\triangle$ sopB1686 & This work \\
\hline rSC0018 & C500 $\triangle a s d A 33$ & This work \\
\hline Streptococcus suis serotype 2 & Wild-type, virulent, CVCC3928 & Lab stock \\
\hline Streptococcus suis serotype 7 & Wild-type isolated in field & Lab stock \\
\hline \multicolumn{3}{|l|}{ Plasmids } \\
\hline pYA3493 & Plasmid Asd + ; pBR ori, $\beta$-lactamase signal sequence-based periplasmic secretion plasmid & {$[46]$} \\
\hline pET28a & expression vector, T7 promoter; $\mathrm{Km}^{r}$ & Novagen \\
\hline pRE112 & oriT oriv sacB Cm ${ }^{R}$ & {$[34]$} \\
\hline pS006 & suicide vector for $\triangle$ sopB1686 & This work \\
\hline pYA3736 & suicide vector for $\triangle a s d A 33$ & pRE112 \\
\hline pET28a-saoA & expression vector for purification of Hig-tag SaoA, T7 promoter, Km+ & pET28a \\
\hline pS-SaoA & pYA3493 with SaoA & pYA3493 \\
\hline
\end{tabular}




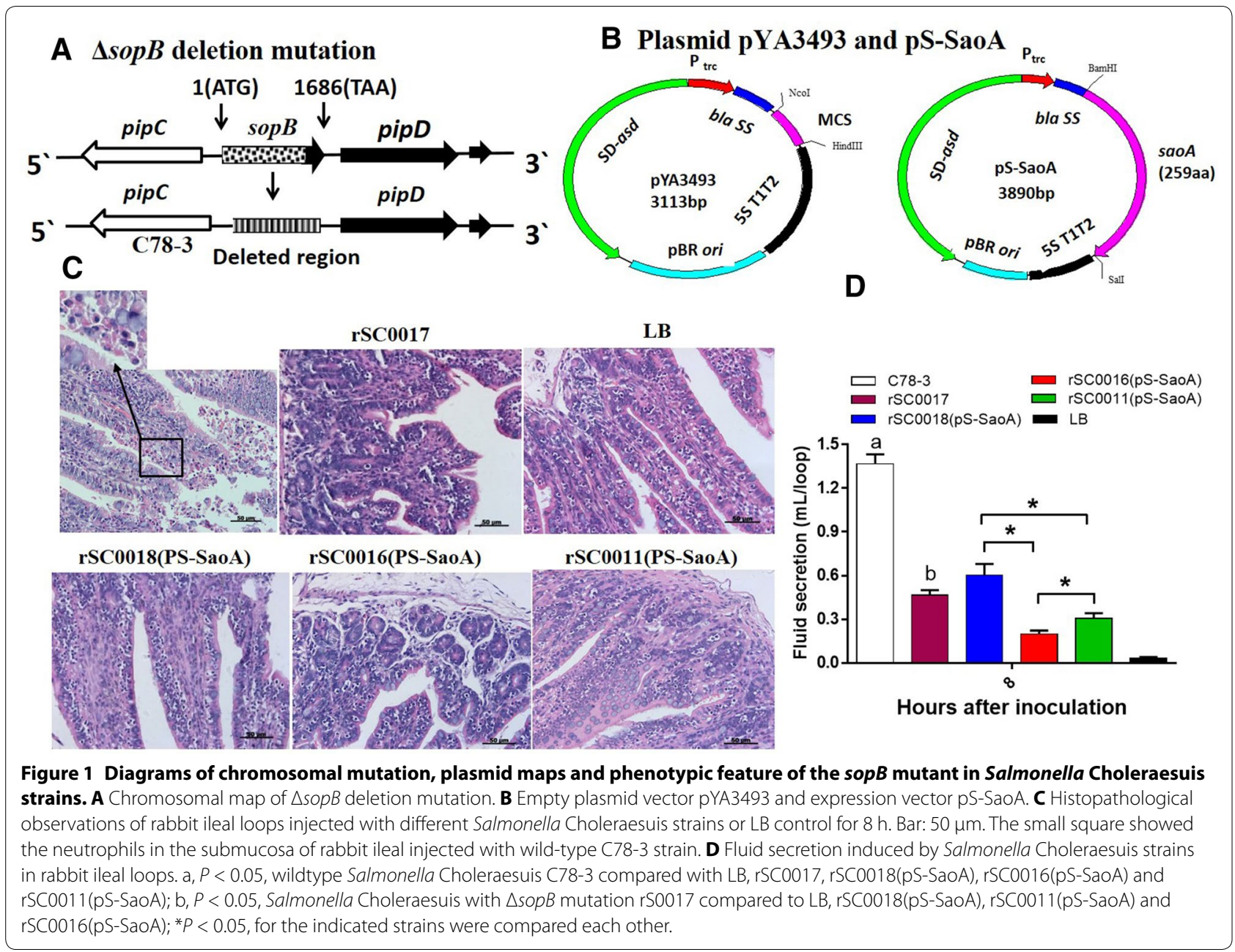

compared as previously described [41]. Briefly, 38-weekold New Zealand White rabbits were fasted overnight and anesthetized with isoflurane by an endotracheal tube. The ileum was exposed and ligated into several loops of 5-6 cm long with $1 \mathrm{~cm}$ spacers. $1 \mathrm{~mL}$ of indicated strains, containing around $1 \times 10^{9}$ colony-forming units (CFU), were injected into separate loops. LB medium was injected into one of the loops as a control. The abdominal musculature was closed using 3-0 chromic gut sutures and the skin closed with 3-0 ethilon sutures. Rabbits were maintained in a thermal blanket at $37{ }^{\circ} \mathrm{C}$. After $8 \mathrm{~h}$, the rabbits were euthanized with an overdose of sodium pentobarbital. The abdomen was reopened and the fluid within the ligated loops was collected and measured. The loops were fixed in $10 \%$ formalin and subjected to histopathological examination.

All mutations were verified by PCR using corresponding primers. The $\Delta \mathrm{P}_{\text {crp527 }}:$ TT araC $\mathrm{P}_{\mathrm{BAD}}$ crp deletion-insertion mutation was confirmed by growing on MacConkey maltose agar with or without $0.2 \%$ arabinose
[29]. LPS profiles were examined by silver staining in $12 \%$ sodium dodecyl sulfate-polyacrylamide gel for $\Delta p m i$ mutation [34]. LacI production for $\Delta$ relA::araC $\mathrm{P}_{\mathrm{BAD}}$ lacl TT mutation was confirmed by Western blot [34]. The $\triangle a s d A$ mutation was confirmed by growth with or without DAP in LB media [34].

\section{Construction and analysis of the plasmid stability of the attenuated Salmonella Choleraesuis vector with plasmids pYA3493 and pS-SaoA}

Primers for $S$. suis saoA gene were designed according to GenBank accession no. GI:8151617 (saoAF:ATGGATCCCAACCTGATGGGGGAC; saoA-R: GCGTCGACCATTGCTTCCTTAGAG) to amplify $\mathrm{N}$-terminal (121-1593 bp) of saoA gene [42]. The DNA of $s a o A$ either from SS2 or SS7 was amplified by PCR, purified by AxyPrep TM PCR Cleanup kit (Axygen, Union, CA, USA), and cloned into the expression vector pET28a to generate plasmid pET28a-SaoA according to the manufacturer's instructions (Novagen, Darmstadt, 
Germany). The identity of the insert in pET28a was verified by DNA sequence analysis. Following digestion of plasmids pET28a-SaoA and pYA3493 with BamHI and SalI, the saoA from SS2 was cloned into pYA3493 to generate $\mathrm{pS}-\mathrm{SaoA}$. pS-SaoA and empty vector pYA3493 were transformed into the live attenuated Salmonella Choleraesuis (Figure 1B).

To evaluate the production of SaoA in live attenuated Salmonella Choleraesuis, sodium dodecyl sulfate polyacrylamide gel electrophoresis (SDS-PAGE) was performed as described [33]. Strains harboring plasmid pS-SaoA or control vector pYA3493 were grown in LB medium with indicating supplements $(0.2 \%$ arabinose and mannose) at $37^{\circ} \mathrm{C}$ with aeration. When the culture reached an $\mathrm{OD}_{600}$ of 0.8 , Isopropyl-d-thiogalactopyranoside (IPTG) was added to the culture, and the culture continued to grow for $3 \mathrm{~h}$. The bacteria numbers were normalized by $\mathrm{OD}_{600} .1 \mathrm{~mL}$ of culture was collected for Western blot analysis using anti-SaoA antiserum as previously described [33]. Briefly, total protein samples were separated on a $10 \%(\mathrm{wt} / \mathrm{vol})$ SDS-PAGE gel and transferred onto nitrocellulose membranes. Phosphate buffered saline (PBS) with 5\% fat-free milk powder and 0.05\% Tween 20 (PBS-T) was used for blocking. The membrane was incubated with an appropriate anti-rabbit polyclonal antibody (1:10 000, anti-SaoA) or anti-GroEL (Sigma, St. Louis, MO, USA) for $1 \mathrm{~h}$ at room temperature, washed three times with PBS-T, and then horseradish peroxidase-conjugated goat anti-rabbit immunoglobulin $\mathrm{G}$ was used as the secondary antibody (Sigma). The membrane was developed with a chemiluminescent substrate using the kit ECL Plus Western Blotting System (GE Healthcare, Chalfont St Giles, UK) according to manufacturer's instructions. Densitometry was quantified using Image J software (Image J2) [43].

To examine the stability of plasmids pS-SaoA and pYA3493 in Salmonella Choleraesuis vector, the strains containing pS-SaoA or pYA3493 were cultured with the daily passage of 1:1000 dilutions for 5 consecutive days (about 50 generations) in LB medium with DAP, arabinose, and mannose. At the 50th generation, $\mathrm{Asd}^{+}$plasmids pS-SaoA and pYA3493 were tested by endonuclease digestion. The mutations of the live attenuated Salmonella Choleraesuis vector were confirmed by PCR. SaoA production was evaluated by Western blot.

\section{Preparation the SaoA protein of SS2 or SS7, antiserum to the SaoA protein of SS2, and Salmonella outer membrane proteins (SOMPs) of Salmonella Choleraesuis}

Escherichia coli strain BL21 carrying pET28a-SaoA was used for the synthesis of the His-tagged SaoA fusion protein (Table 1$)$. Cells were grown to mid-log phase $\left(\mathrm{OD}_{600}\right.$ of 0.6) in LB medium with kanamycin at $37{ }^{\circ} \mathrm{C}$ and induced with $1 \mathrm{mM}$ IPTG for $3 \mathrm{~h}$. The His-tagged SaoA protein was purified by using a CelLytic B Plus kit (Sigma) and a His-Select nickel affinity gel (Sigma) according to manufacturer's instructions. To create rabbit antibodies to SaoA, two female New Zealand White rabbits (8 weeks old) were injected subcutaneously with an emulsion consisting of a 1:1 ratio of $400 \mu \mathrm{g}$ of the His-tagged SaoA protein to complete Freund adjuvant (Sigma). Two weeks after the primary injection, the immunization was repeated but with incomplete Freund adjuvant (Sigma) instead of complete adjuvant. Three weeks after the second immunization, the rabbits were boosted with $200 \mu \mathrm{g}$ of the His-tagged SaoA protein without adjuvant. Two weeks after the third immunization, the rabbits were bled to obtain anti-SaoA rabbit antiserum [34].

Salmonella outer membrane proteins (SOMPs) were prepared from wild-type Salmonella Choleraesuis C783. Briefly, total envelope pellets were suspended in $4 \mathrm{~mL}$ of $20 \mathrm{mM}$ Tris $-\mathrm{HCl}(\mathrm{pH}$ 8.6) containing 1\% Sarkosyl and incubated for $30 \mathrm{~min}$ on ice. The outer membrane fraction was obtained as a pellet after centrifugation at $132000 \times g$ at $4{ }^{\circ} \mathrm{C}$ for $1 \mathrm{~h}$. The pellet was re-suspended in $4 \mathrm{~mL}$ of $20 \mathrm{mM}$ Tris- $\mathrm{HCl}$ buffer $(\mathrm{pH} \mathrm{8.6)}$ and stored at $-20^{\circ} \mathrm{C}$.

\section{Distribution of Salmonella bacteria in BALB/c mice}

Colonization assay for mutant strain was carried out as described [34]. 140 7-week-old female BALB/c mice were divided into seven groups with 20 mice per group. Groups of mice were orally inoculated with $20 \mu \mathrm{L}$ buffered saline with gelatin (BSG) containing $1 \times 10^{9} \mathrm{CFU}$ of Salmonella strains. Liver, spleen, and Peyer's patches of mice were collected on days $3,7,14$, and 21 post-infection. Tissues were weighed and homogenized in a final volume of $1 \mathrm{~mL}$ BSG [39]. Serial dilutions were plated onto MacConkey agar plates containing $1 \%$ lactose, with or without $0.2 \%$ arabinose and $0.2 \%$ mannose, to determine the number of viable bacteria. Plates were incubated at $37{ }^{\circ} \mathrm{C}$ for at least $18 \mathrm{~h}$. The residual $900 \mathrm{~mL}$ of homogenized tissues were inoculated into $5 \mathrm{~mL}$ Tetrathionate Broth (Difco) for Salmonella enrichment when no colonies were observed on the plates. Samples that were negative by direct plating and positive by enrichment were recorded as $10 \mathrm{CFU} / \mathrm{g}$. Samples that were negative by both direct plating and enrichment were recorded as $0 \mathrm{CFU} / \mathrm{g}$ [39]. The assay was performed twice, and the data were pooled.

\section{Immunization of mice and piglets Immunization of mice}

Salmonella Choleraesuis vector strains harboring plasmids pS-SaoA or pYA3493 (empty vector) were grown in LB broth with $0.2 \%$ arabinose and $0.2 \%$ mannose 
overnight at $37^{\circ} \mathrm{C}$ as standing cultures that were diluted 1:100 in the same medium. The culture was grown with aeration $(180 \mathrm{rpm})$ at $37{ }^{\circ} \mathrm{C}$ to an $\mathrm{OD}_{600}$ of $0.85-0.9$. Bacteria were collected by centrifugation at room temperature and re-suspended in BSG. 15 7-week-old $\mathrm{BALB} / \mathrm{c}$ female mice were inoculated orally with $20 \mu \mathrm{L}$ BSG containing $1 \pm 0.3 \times 10^{9}$ CFU of Salmonella Choleraesuis vectors with $\mathrm{pS}-\mathrm{SaoA}$ or plasmid pYA3493 or 20 $\mu \mathrm{L}$ BSG control. Mice were boosted with the same dose of the same strain 3 weeks later. About $100 \mu \mathrm{L}$ of whole blood was obtained by mandibular vein puncture 3 weeks after primary inoculation and 2 weeks after boosting. Serum was removed from the whole-blood samples and stored at $-20{ }^{\circ} \mathrm{C}$. Vaginal-wash samples in mice were collected at the indicated time and stored at $-20{ }^{\circ} \mathrm{C}$ as described [33, 34]. Ten mice from each group were challenged with SS2, and five mice were used for cytokine detection. This experiment was performed twice, with each group (15 mice) receiving approximately the same dose of vaccine. The results from both experiments were similar and the data were pooled for analysis.

\section{Immunization of piglets}

The preparation of the inoculum of Salmonella Choleraesuis vector strains harboring plasmids $\mathrm{pS}$-SaoA or pYA3493 was performed as described in a previous section. Seventy-five 3-week-old castrated piglets were purchased from a commercial pig farm in Jiangsu Province, China. To select the pigs with negative antibody titers against SS2, SS7, and Salmonella Choleraesuis, blood was obtained from pigs by cranial vena cava and incubated at $37^{\circ} \mathrm{C}$ for $60 \mathrm{~min}$. The resulting clot was pelleted by centrifugation. Serum was removed from the whole-blood sample and stored at $-20{ }^{\circ} \mathrm{C}$. Serum IgG responses to SS2 SaoA, SS7 SaoA, and Salmonella Choleraesuis outer membrane proteins (OMPs) were measured by enzymelinked immunosorbent assay (ELISA) (see below) [33, $44,45]$. The IgG titer that was less than cut-off value was considered as seronegative. These piglets were randomly assigned to five groups with 15 piglets/group, named rSC0016(pS-SaoA) group, rSC0016(pYA3493) group, rSC0018(pS-SaoA) group, rSC0018(pYA3493) group, and BSG group. Piglets were inoculated orally with $10 \mathrm{~mL}$ $1 \pm 0.3 \times 10^{9} \mathrm{CFU}$ of Salmonella Choleraesuis vector strains carrying either the SaoA expression plasmid pSSaoA or empty plasmid pYA3493 or BSG control. Piglets were boosted with the same dose of the same strain 3 weeks later. Serum and nasal swabs were collected at 3 , and 5 weeks after first immunization $[10,16]$. In each group, five piglets were challenged with SS2 and five with SS7 at 2 weeks after a boost. Five piglets were used to detect cytokine levels in the blood and spleen at different time points $(0.5,3,5$, and 7 days after boost). This experiment was performed twice with each group receiving approximately the same dose of vaccine. The results from both experiments were similar and the data were pooled for analysis.

\section{ELISA test}

Serum IgG responses to SaoA, to OMPs of Salmonella Choleraesuis in mice and pigs, and vaginal wash IgA antibody to SaoA in mice and nasal cavity wash IgA in pigs against SaoA were measured by ELISA. The initial dilutions of serum or vaginal/nasal wash from an individual animal were $1: 50$ or $1: 10$, respectively. Briefly, $100 \mu \mathrm{L}$ solutions containing either $1 \mu \mathrm{g} /$ well of Salmonella OMPs or of SaoA in sodium carbonate-bicarbonate coating buffer ( $\mathrm{pH}$ 9.6) was used to coat Nunc-Immuno MaxiSop 96-well plates (Corning, NY, USA). The plates were then incubated overnight at $4{ }^{\circ} \mathrm{C}$. The next day, the plates were washed 3 times with PBST (PBS with $0.1 \%$ Tween 20) and then blocked with a PBST containing $2 \%$ BSA solution for $2 \mathrm{~h}$ at room temperature. A $100 \mu \mathrm{L}$ volume of serially diluted sample was added in triplicate to individual wells, and the plates were incubated for $1 \mathrm{~h}$ at room temperature. After washing wells with PBST, goat anti-mouse or goat anti-pig IgG-horseradish peroxidase (HRP) detection antibody (Sigma, St. Louis, MO, USA) diluted 1:5000 in PBST was added to the wells and the plate was incubated at room temperature for $60 \mathrm{~min}$ with gentle rocking. Plates were developed with 1-Step TM Ultra TMB-ELISA (Thermo Fisher Scientific, Waltham, MA, USA) and quenched with $3 \mathrm{~N}_{2} \mathrm{SO} 4$. Absorbance was recorded at $450 \mathrm{~nm}$ using an automated ELISA plate reader (model EL311SX; Biotek, Winooski, VT). Absorbance readings 2.1 times higher than the baseline values of preimmune sera (negative control: about 0.045-0.052) were considered positive [33, 44-46].

Sera were collected from mice or piglets on $0.5,3$, and 5 days. In addition, spleen tissues were removed at 7 days after boost immunization. Spleens were homogenized in a final volume of $1 \mathrm{~mL}$ (mouse tissues) or $5 \mathrm{~mL}$ (pig tissues) BSG at a ratio of weight: volume of 1:9. The supernatants were stored at $-70{ }^{\circ} \mathrm{C}$. Cytokines in sera and spleen of mice or pigs were analyzed by sandwich ELISA using commercial kits (Abcam, Cambridge, UK, except pig-specific IL4 ELISA kit, which was purchased from Thermo Fisher Scientific) according to manufacturer's protocols.

\section{Analysis of the cross-reactivity between SS2 and SS7 in pigs}

Since the $s a o A$ gene was from SS2, the cross-reactivity of sera from pigs immunized with rSC0018(pS-SaoA) or rSC0016(pS-SaoA) was checked against total cell lysates from SS2 or SS7, respectively. Total S. suis proteins (SS2 
or SS7 strain) were extracted from bacterial culture and $10 \mu \mathrm{g}$ of proteins were separated by $12 \%$ SDS-PAGE gel electrophoresis and transferred to nitrocellulose membrane. The membrane was incubated with immunized pig serum (1:100 dilution) for $1 \mathrm{~h}$ at room temperature and subsequently washed three times with $0.05 \% \mathrm{w} / \mathrm{v}$ Tween-20 in TBS for $5 \mathrm{~min}$. Anti-pig IgG conjugated to horseradish peroxidase (1:5000 dilution, Sigma) was used as secondary antibodies. The membrane was developed with a chemiluminescent substrate using the kit ECL Plus Western Blotting System (GE Healthcare) according to manufacturer's instructions.

\section{Challenge experiment with SS2 in mice or SS2 and SS7 in pigs}

Ten mice were challenged with $3 \times 10^{8} \mathrm{CFU}$ of SS2 intraperitoneally (i.p.) at 2 weeks after the booster. The $50 \%$ lethal dose $\left(\mathrm{LD}_{50}\right)$ of SS2 in BALB/c mice was $1.2 \times 10^{7}$ CFU. Challenged mice were monitored daily for 15 days [33].

Five pigs in each group were challenged with SS2 by intravenous injection (i.v.) with $5.0 \times 10^{8} \mathrm{CFU}\left(\mathrm{LD}_{50}\right.$ of SS2 in pigs was $\left.5.6 \times 10^{7} \mathrm{CFU}\right)$. The clinical symptoms and survival of pigs were monitored for 14 days. When needed, the brain of pigs was fixed in $10 \%$ formalin buffer ( $\mathrm{pH}$ 7.2). Thin sections (5 mm) of tissue were stained with hematoxylin/eosin and examined by light microscopy to detect histological changes caused by SS2. Another five pigs in each group were challenged by i.v. with $1.0 \times 10^{10}$ CFU of SS7. The rectal temperatures of pigs challenged with SS7 were measured at $0,1,2,3,4,5,6$, and 7 days post-inoculation [47].

\section{Statistical analysis}

Data were presented as the geometric means and standard deviations for all assays. The Mann-Whitney U test (GraphPad Software, Inc., San Diego, CA) was used to evaluate the persistence of strains in vivo and antibody levels of mice. The Kaplan-Meier method (SPSS software) was applied to obtain the survival fractions following i.p. challenges of immunized mice and i.v. challenges of immunized pigs. A $P$ value of 0.05 was considered statistically significant.

\section{Results}

\section{Construction of sopB deletion in Salmonella Choleraesuis} vaccine vectors

Previous reports have shown that the inactivation of $\operatorname{sop} B$ in Salmonella Typhimurium improves humoral and cellular immunity in the host $[35,48]$. To improve the safety and increase the immunogenicity of Salmonella Choleraesuis vector, a $\operatorname{sop} B$ mutation was introduced into wildtype Salmonella Choleraesuis C78-3 to generate strain rSC0017 (Figure 1A) or into Salmonella Choleraesuis
rSC0013 to generate strain rSC0016 (Table 1). Strain rSC0016 has the mutations for the regulated delayed attenuation system and the regulated delayed antigen synthesis system, characterized by $\Delta \mathrm{P}_{\text {crp }}:$ TT araC $\mathrm{P}_{\mathrm{BAD}}$ crp, $\triangle p m i$, and $\triangle$ relA::ara $C \mathrm{P}_{\mathrm{BAD}}$ lacI TT [34], in addition to the $\operatorname{sop} B$ mutation. It also has a $\triangle a s d A$ mutation for the balanced-lethal system to facilitate its use as a vector.

\section{Phenotypic characterization of live attenuated Salmonella Choleraesuis vaccine vector with sop $B$ mutation}

SopB plays a role in water efflux, fluid secretion, and subsequent diarrhea associated with Salmonella infection [49]. In this study, the vaccine strains $\mathrm{rSC0018(pS-}$ SaoA), rSC0011(pS-SaoA), rSC0016(pS-SaoA), the strain rSC0017 with a $\operatorname{sop} B$ deletion mutation, the parental wild-type strain C78-3, and a LB control were injected into six parts of the ileal loop in rabbits to detect the effect of the sopB deletion in Salmonella Choleraesuis. Hematoxylin-eosin staining of ileal loop sections revealed populations of neutrophilic cells in the ileum loops infected by the wild-type strain C78-3, but not in those infected by rSC0017, rSC0018(pS-SaoA), rSC0011(pS-SaoA), or rSC0016(pS-SaoA). These loops were similar to the loops injected by LB (Figure 1C). The fluid accumulation in the ileal loops injected with the wild-type parental strain C78-3 was significantly higher than those injected with rSC0017, rSC0018(pS-SaoA), rSC0011(pS-SaoA), rSC0016(pS-SaoA) and LB (Figure 1D). Particularly, the inflammatory exudate in the ileal loops injected with rSC0016(pS-SaoA) containing sop $B$ mutation was significantly reduced compared to that injected with $\mathrm{rSC} 0011(\mathrm{pS}-\mathrm{SaoA})$ without the $\operatorname{sop} B$ mutation. In addition, the fluid accumulation in the ileal loops injected with rSC0018(pS-SaoA) was significantly more than that in those injected with rSC0011(pS-SaoA) and $\mathrm{rSC} 0016(\mathrm{pS}-\mathrm{SaoA})$, even higher than that of rSC0017 (Figure 1D). Little fluid was detected in the loops injected with LB control, which was significantly less than those injected with C78-3, rSC0017, SC0018(pS-SaoA), rSC0011(pS-SaoA), and rSC0016(pS-SaoA) (Figure 1D).

The phenotype of $\Delta \mathrm{P}_{\text {crp527 }}: \mathrm{TT}$ araC $\mathrm{P}_{\mathrm{BAD}}$ crp mutation was tested on MacConkey maltose agar with and without arabinose. The strain with $\Delta \mathrm{P}_{\text {crp527 }}: \mathrm{TT}$ araC $\mathrm{P}_{\mathrm{BAD}}$ crp mutation formed red colonies on MacConkey maltose agar in the presence of arabinose, and white colonies in the absence of arabinose (Figure 2A) [29, 46]. The smooth LPS pattern was observed in strain rSC0016 grown with mannose, and rough without mannose due to the presence of $\Delta p m i$ mutation (Figure 2B) [34]. To verify the regulated delayed synthesis of heterologous protein, strain rSC0016(pS-SaoA) was passaged four times in arabinose -and mannose-free medium after it was grown in nutrient broth medium with $0.2 \%$ arabinose 


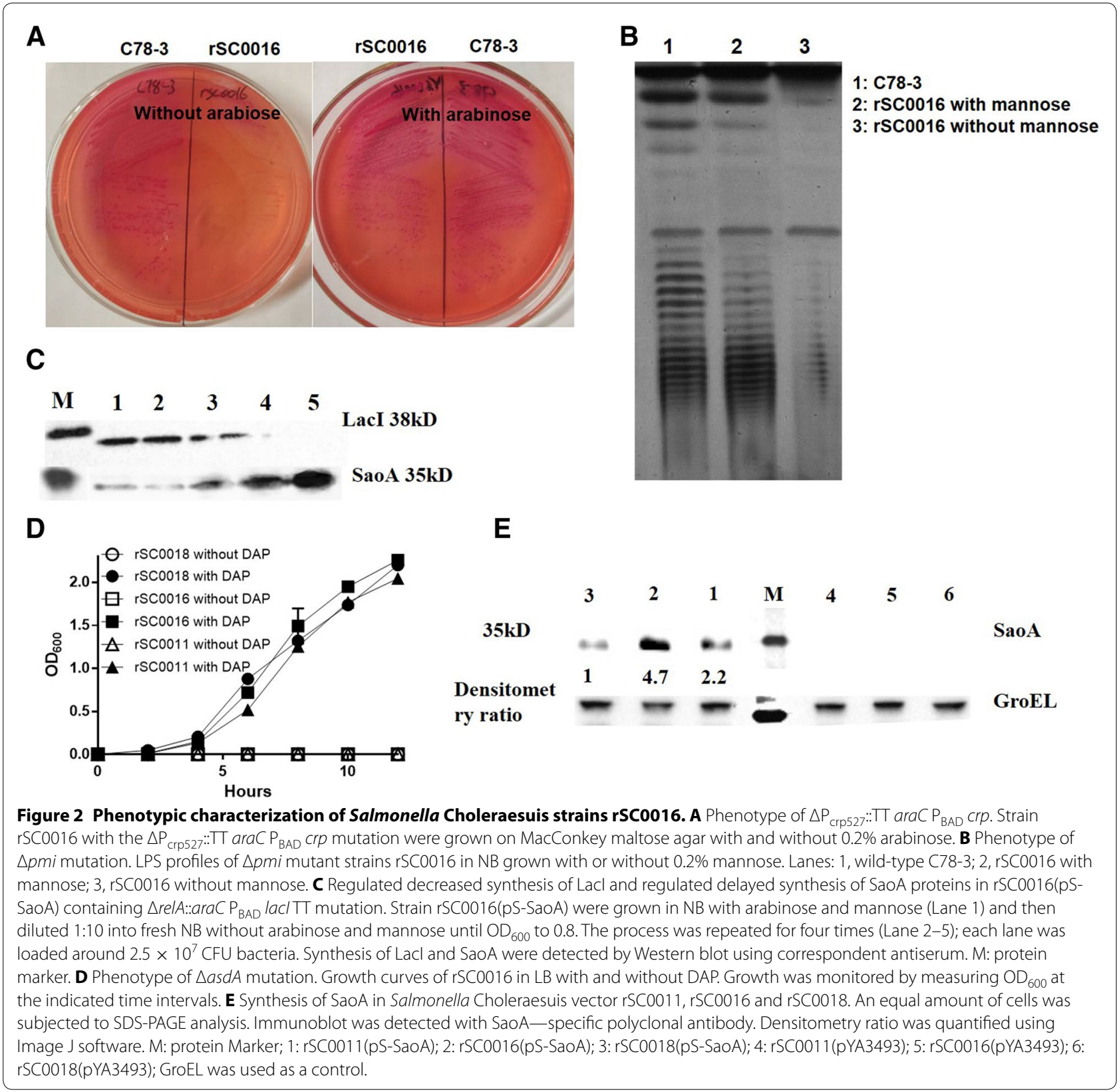

and mannose, as described previously [34]. The expected phenotype, the gradual disappearance of the LacI protein and the increased production of the heterologous protein SaoA, was observed in the strain rSC0016(pS-SaoA) (Figure 2C) [29, 46]. The introduction of the $\triangle a s d A$ mutation into Salmonella Choleraesuis strains rSC0010, rSC0013, and C500 (Table 1) $[46,50]$ did not affect the growth of the resulting strains rSC0011, rSC0016, and rSC0018 (Figure 2D). However, the production of SaoA protein in rSC0011 and rSC0016 was 2.2-fold and 4.7fold higher than that in $\mathrm{rSC0018}$, respectively, whereas the production of SaoA protein in strain $\mathrm{rSC} 0016$ was 2.5 -fold higher than that in strain $\mathrm{rSC} 0011$ (pS-SaoA) (Figure 2E).

\section{Plasmids pYA3493 and pS-SaoA are stable in attenuated Salmonella Choleraesuis rSC0016 strain}

A single colony of rSC0018, rSC0016 and rSC0011 either with pS-SaoA or pYA3493 was grown in LB with $0.2 \%$ mannose, $0.2 \%$ arabinose, and $50 \mu \mathrm{g} / \mathrm{mL}$ DAP for 50 generations [34]. All the colonies that were examined using PCR or endonuclease digestion contained either 


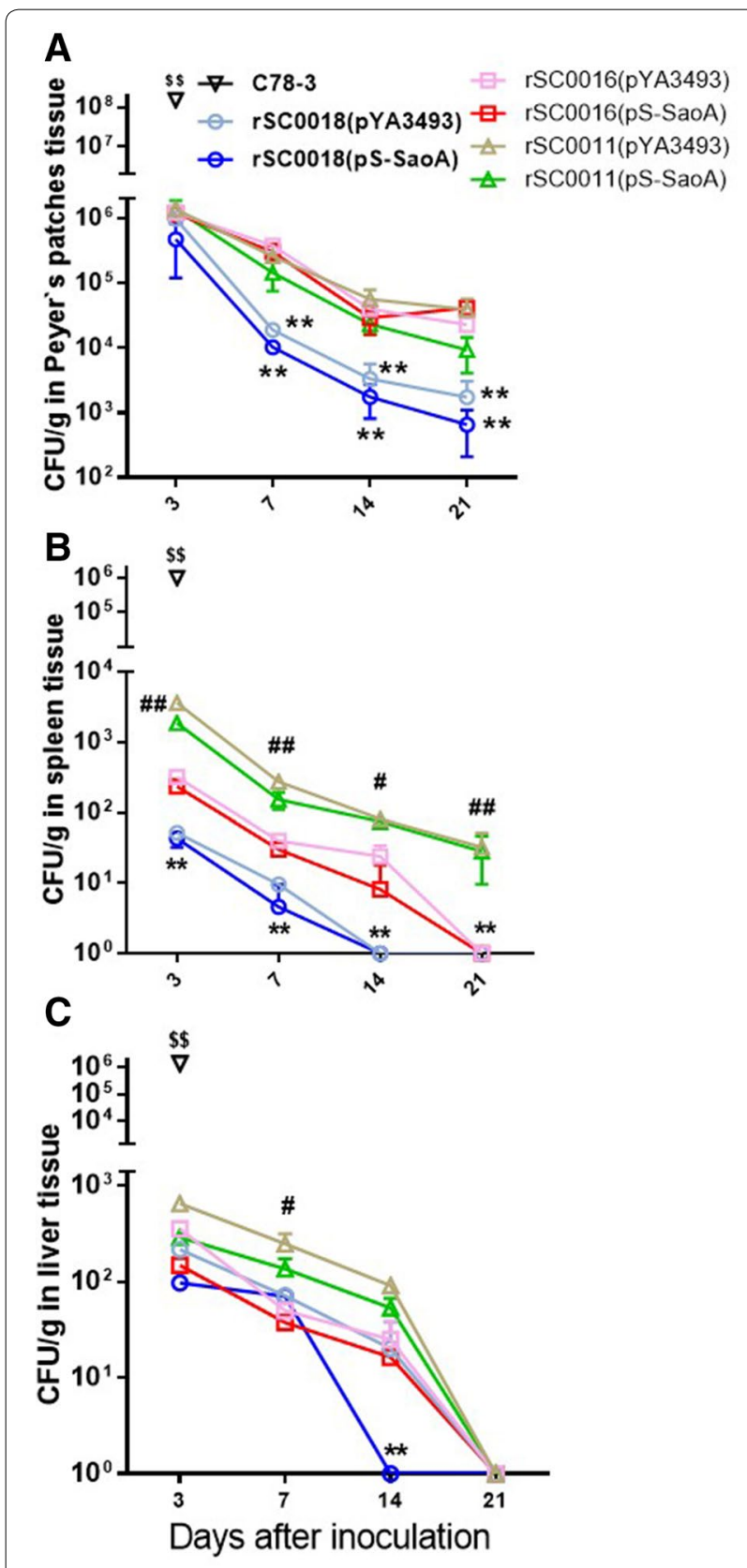

Figure 3 Colonization of Salmonella Choleraesuis rSC0016 in BALB/c mice at different time points. The numbers of Salmonella Choleraesuis C78-3, rSC0018(pYA3493), rSC0018(pSSaoA), SC0016(pYA3493), rSC0016(pS-SaOA), rSC0011 (pYA3493), and SC0011 (pS-SaoA) in Peyer's patches (A), spleen (B) and liver (C), of mice at 3, 7, 14, and 21 days after oral inoculation with $1.0 \pm 0.3 \times 10^{9} \mathrm{CFU}$ of the indicated strains were plotted. Bars represent the arithmetic mean \pm standard deviations from ten mice per group. ${ }^{*} P<0.01$, for rSC0018 compared to rSC0016 or to rSC0011 with either pYA3493 or pS-SaOA, respectively; ${ }^{\#} P<0.05,{ }^{\# \#} P<0.01$, for rSC0011 compared to rSC0016 carrying either pYA3493 or pS-SaoA, respectively; $\$ \$<0.01$, for C78-3 compared to rSC0018, rSC0016 and rSC0011 carrying either pYA3493 or pS-SaoA, as indicated. The data were collected from two independent experiments. plasmid pS-SaoA or plasmid pYA3493, indicating that pS-SaoA and pYA3493 are equally stable in the vaccine strains (data not shown). These colonies can produce the expected SaoA protein (data not shown).

\section{Distribution of Salmonella Choleraesuis rSC0016 vector in BALB/c mice}

The Salmonella Typhimurium $\triangle \operatorname{sop} B$ mutant is attenuated in its ability to cause gastroenteritis in calves [51], but not in its invasion of host tissues [52]. The distributions of wild-type Salmonella Choleraesuis C78-3, rSC0018(pYA3493), rSC0018(pSSaoA), rSC0016(pYA3493), rSC0016(pS-SaoA), rSC0011(pYA3493), and rSC0018(pS-SaoA) in the Peyer's patches, spleens, and livers of $\mathrm{BALB} / \mathrm{c}$ mice were compared. The mice inoculated with wildtype Salmonella Choleraesuis C78-3 died 3 days after inoculation, whereas the mice infected orally with $10^{9}$ CFU of rSC0018(pYA3493), rSC0018(pSSaoA), rSC0016(pYA3493), rSC0016(pS-SaoA), rSC0011(pYA3493), or rSC0011(pS-SaoA) survived without displaying any disease symptom. The bacteria titer of wild-type strain C78-3 in Peyer's patches, spleen, and liver were significantly higher than those of strains rSC0016(pYA3493), rSC0016(pSSaoA), rSC0011(pYA3493), rSC0011(pS-SaoA), rSC0018(pYA3493), and rSC0018(pS-SaoA) at 3 days after inoculation (Figures $3 \mathrm{~A}-\mathrm{C}$ ). The three attenuated strains rSC0011, rSC0016, and rSC0018 with plasmid pS-SaoA or pYA3493 colonized Peyer's patches equally well 3 days after infection, suggesting that the deletion of $\operatorname{sop} B$ did not affect the initial colonization of Peyer's patches by Salmonella Choleraesuis vaccines. The titers of bacteria in Peyer's patches were similar for strains rSC0016(pYA3493), rSC0016(pSSaoA), rSC0011(pYA3493), and rSC0016(pS-SaoA) at 3-21 days post-inoculation. All of these strains colonized Peyer's patches better than attenuated vaccine strains rSC0018(pYA3493) and rSC0018(pS-SaoA) did at 7, 14, and 21 days post-inoculation, with about 13-fold, 22.5-fold, and 60-fold higher titers, respectively (Figure 3A). In the spleen, the titer of bacteria of strains rSC0011(pYA3493) and rSC0011(pS-SaoA) was significantly higher than that of rSC0016(pYA3493), rSC0016(pS-SaoA), rSC0018(pYA3493), and rSC0018(pS-SaoA), respectively, at $3,7,14$, and 21 days, respectively (Figure 3B). Furthermore, more rSC0016(pYA3493), rSC0016(pS-SaoA) colonized in spleen than that rSC0018(pYA3493) and rSC0018(pSSaoA) did at 3, 7, and 14 days, respectively (Figure $3 \mathrm{~B})$. These results indicated that the $\operatorname{sop} B$ mutation reduced the colonization ability of Salmonella Choleraesuis vaccine strains in mice spleen. Salmonella 
vectors $\mathrm{rSC} 0016$ and $\mathrm{rSC} 0011$ with the regulated delayed strategy displayed better colonization in the spleen compared with rSC0018. In liver, the titers of rSC0016(pYA3493) and rSC0016(pS-SaoA) were similar to those of rSC0011(pYA3493), rSC0011(pS-SaoA), rSC0018(pYA3493), and rSC0018(pS-SaoA) at 3 days after inoculation (Figure $3 \mathrm{C}$ ). At day 7 post-inoculation, the titers of rSC0016(pYA3493) and rSC0016(pSSaoA) were 5.0-fold and fourfold lower than those of rSC0011(pYA3493) and rSC0011(pS-SaoA), respectively, whereas the strain $\mathrm{rSC} 0018(\mathrm{pS}-\mathrm{SaoA})$ was the fastest to be cleared in liver (Figure $3 \mathrm{C}$ ). These results indicated that the $\operatorname{sop} B$ deletion slightly impaired the colonization in the liver of Salmonella Choleraesuis vaccine strains. Although the titers of rSC0011(pS-SaoA) and rSC0016(pS-SaoA) were slightly lower than those of rSC0011(pYA3493) and rSC0016(pYA3493) in Peyer's patches, spleen, and liver, they were not significantly different (Figures $3 \mathrm{~A}-\mathrm{C}$ ).

\section{Salmonella Choleraesuis vaccine vector with sopB mutation} induces higher antibody responses in mice

To test the immunogenicity of Salmonella Choleraesuis vaccine strain that combines regulated delayed strategy and the $\operatorname{sop} B$ mutation, the antibody responses to SS2 SaoA and Salmonella Choleraesuis OMPs in mice were measured at 3 and 5 weeks after the primary immunization (Figures $4 \mathrm{~A}, \mathrm{~B}$ and $\mathrm{C}$ ). Higher serum IgG and vaginal IgA titers against SaoA were detected in the mice immunized with the live attenuated Salmonella Choleraesuis vaccines carrying plasmid pSSaoA [rSC0016(pS-SaoA), rSC0011(pS-SaoA), and rSC0018(pS-SaoA)] compared to those with BSG or the strain containing the empty vector pYA3493 (Figures $4 \mathrm{~A}$ and $\mathrm{B}$ ). The levels of serum IgG against SaoA in mice immunized with $\mathrm{rSC} 0016(\mathrm{pS}$-SaoA) were similar to those in mice immunized with rSC0011(pSSaoA), yet significantly higher than those immunized with rSC0018(pS-SaoA) (Figure 4A). Higher vaginal IgA titers against SaoA were observed in the mice immunized with rSC0016(pS-SaoA) or rSC0011(pSSaoA) compared to those with rSC0018(pS-SaoA) (Figure $4 \mathrm{~B}$ ). At 3 or 5 weeks after vaccination, the mucosal IgA responses against SaoA in mice immunized with rSC0016(pS-SaoA) were also significantly higher than those with rSC0011(pS-SaoA) (Figure 4B). At 3 weeks after the primary immunization, the IgG antibody responses to Salmonella Choleraesuis OMPs were significantly higher in mice immunized with rSC0016(pS-SaoA) than those with rSC0011(pS-SaoA) or rSC0018(pS-SaoA) (Figure 4C). At 5 weeks postinfection, higher IgG responses against Salmonella Choleraesuis OMPs were observed in mice immunized with rSC0016 carrying either pS-SaoA or pYA3493 than those with rSC0011 or rSC0018 (Figure 4C).

Salmonella Choleraesuis vaccine vector with sopB mutation induces higher interferon $\gamma$ (IFN- $\gamma$ ), interleukin 4 (IL-4), and interleukin 17A (IL-17A) responses in mice

A previous study showed that the introduction of a $\operatorname{sop} B$ mutation into different attenuated Salmonella Typhimurium strains enhanced the immune responses to the delivered heterologous antigen in mice [48]. To further characterize the influence of $\operatorname{sop} B$ on immune responses, the levels of IFN- $\gamma$, IL-4, and IL-17A in mice sera collected at $0.5,3$, and 5 days and in the spleen collected at 7 days after the booster were measured. The concentrations of IFN- $\gamma$, IL-4, and IL-17A induced in mice immunized with vector rSC0016, rSC0011, or rSC0018 carrying either pYA3493 or pS-SaoA were significantly higher in the sera and spleen tissues than those induced in mice treated with BSG (Figures $5 \mathrm{~A}-\mathrm{C}, P<0.01$ ). The three vectors that contain $\mathrm{pS}$-SaoA (rSC0011, rSC0016 and rSC0018) induced higher levels of IFN- $\gamma$, IL-4, and IL-17A than the strain with the empty vector pYA3493 in sera and spleen of mice (Figures $5 \mathrm{~A}-\mathrm{C}$, ${ }^{*} \mathrm{P}<0.05$; ** $P<0.01$ ). Conversely, rSC0018(pS-SaoA) induced similar levels of IFN- $\gamma$ and IL-4 in sera compared to those induced by rSC0018(pYA3493) at 3 and 5 days after boost immunization (Figures 5A and B).

Higher IFN- $\gamma$, IL-4, and IL-17A levels were produced in the sera and spleens of mice immunized with rSC0016 or rSC0011 carrying either the empty vector pYA3493 or the SaoA-expressing plasmid pS-SaoA than in those with rSC0018 (Figures 5A-C; ${ }^{\#} P<0.05,{ }^{\# \#} P<0.01$ ). rSC0011 with pYA3493 or pS-SaoA induced similar levels of IL-4 to those of rSC0018 with pYA3493 or pS-SaoA (Figure $5 \mathrm{~B}$ ). Higher levels of IFN- $\gamma$, IL-4, and IL-17A were observed in the sera and spleens of mice immunized with $\mathrm{rSC0016}(\mathrm{pS}-\mathrm{SaoA})$ compared to those in mice immunized with rSC0011(pS-SaoA) (Figures 5A-C; $\left.{ }^{\#} P<0.05,{ }^{\# \#} P<0.01\right)$. Alternatively, the level of IL-17A in the spleens of mice immunized with rSC0016(pSSaoA) was similar to rSC0011(pS-SaoA) (Figure 5C). The levels of IFN- $\gamma$, IL-4, and IL-17A that were induced by rSC0016(pYA3493) were significantly higher than those induced by rSC0011 (pYA3493) in the sera at 3 , and 5 days after the booster, as well as in the spleen (Figures $5 \mathrm{~A}-\mathrm{C}$; $\left.{ }^{\#} P<0.05,{ }^{\#} P<0.01\right)$. These results indicate that the vector rSC0016 with the $\triangle \operatorname{sop} B$ mutation induced enhanced responses more than the rSC0011 strain did [35].

Salmonella Choleraesuis vaccine vector with sopB mutation confers protection against SS2 in mice

When the immunized mice were challenged with 25 times the $\mathrm{LD}_{50}$ of wild-type SS2, $100 \%$ protection 


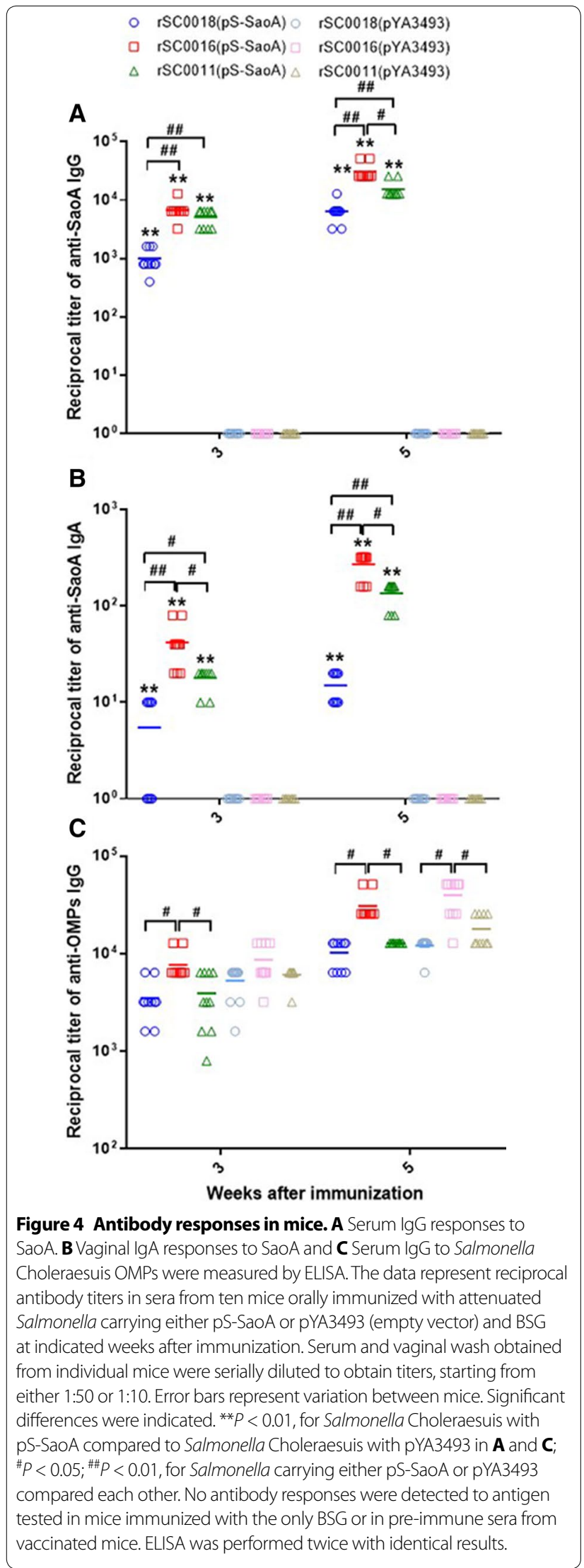

was observed in the mice immunized with strain rSC0016(pS-SaoA), 40\% with strain rSC0011(pS-SaoA), and $0 \%$ with $\mathrm{rSC} 0018(\mathrm{pS}-\mathrm{SaoA})$ (Table 2). All the mice immunized with BSG or the strain containing the empty vector pYA3493 died at 2-3 days after challenge. These results suggested that the $\mathrm{rSC} 0016(\mathrm{pS}-\mathrm{SaoA})$ strain with the $\triangle \operatorname{sop} B$ mutation conferred significantly greater protection than $\mathrm{rSC} 0011(\mathrm{pS}-\mathrm{SaoA})$ or $\mathrm{rSC} 0018(\mathrm{pS}-\mathrm{SaoA})$ (Table $2 ;{ }^{* *} P<0.01$ ).

\section{Salmonella Choleraesuis vaccine vector with sopB mutation induces higher antibody responses in piglets}

The immune responses to $\mathrm{rSC} 0016(\mathrm{pS}-\mathrm{SaoA})$ were tested in the piglets. The antibody responses to SS2 SaoA and Salmonella OMPs were measured at 3 and 5 weeks after the primary immunization (Figures 6A, B and C). Higher serum IgG and mucosal IgA titers against SaoA were observed at 3 and 5 weeks post-immunization in the swine immunized with rSC0016(pS-SaoA) or rSC0018(pS-SaoA) compared to those with BSG or the empty vector pYA3493 (Figures $6 \mathrm{~A}$ and $\mathrm{B} ;{ }^{* *} P<0.01$ ). No antibody responses against SaoA were observed in the swine immunized with rSC0016(pYA3493), rSC0018(pYA3493) (Figures 6A and B), or BSG. Notably, the piglets immunized with rSC0016(pS-SaoA) displayed significantly higher serum IgG and mucosal IgA titers against SaoA compared to those with rSC0018(pSSaoA) (Figures 6A and B; ${ }^{\#} P<0.05,{ }^{\# \#} P<0.01$ ). Similarly, the titers of antibodies directed against Salmonella Choleraesuis OMPs induced in pigs by rSC0016(pSSaoA) or rSC0016(pYA3493) were higher than those by rSC0018(pS-SaoA) or rSC0018(pYA3493), respectively (Figure 6C; ${ }^{\#} P<0.05,{ }^{\# \#} P<0.01$ ), indicating that rSC0016 induces stronger immune responses than did rSC0018.

Salmonella Choleraesuis vaccine vector with sopB mutation induces higher levels of IFN- $\gamma$, IL-4, and IL-17A in piglets To further characterize the immune trends in piglet immunized with strain rSC0016 delivering a heterologous antigen, the levels of cytokines IFN- $\gamma$, IL-4, and IL-17A in the sera of immunized piglets at $0.5,3$, and 5 days, and in the spleens at 7 days after a boost were tested (Figures 7A-C). Significantly higher levels of IFN- $\gamma$, IL-4, and IL-17A were observed in the piglets immunized with the strains rSC0018 and rSC0016 carrying either $\mathrm{pS}-\mathrm{SaoA}$ or the empty vector pYA3493 compared to those with BSG group (Figures 7A-C; $P<0.01)$. Higher levels of IFN- $\gamma$, IL-4, and IL-17A were also detected in the swine immunized with strains rSC0018 and rSC0016 carrying pS-SaoA compared to the strains carrying the empty vector pYA3493 (Figures $7 \mathrm{~A}-\mathrm{C}$; $\left.{ }^{*} \mathrm{P}<0.05,{ }^{* *} \mathrm{P}<0.01\right)$. Conversely, the levels of IFN- $\gamma$ and IL-17A in the sera of swine immunized 

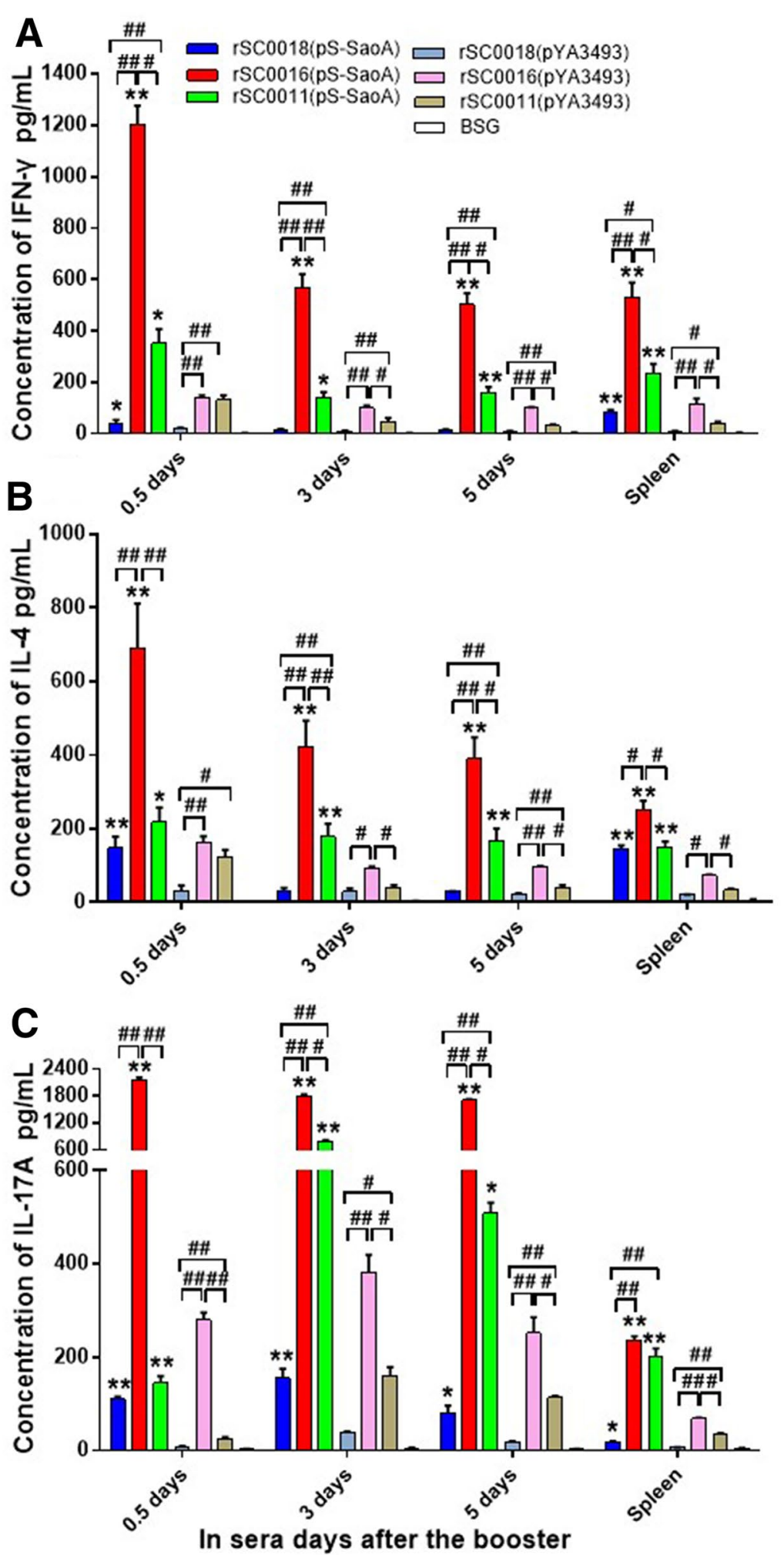

Figure 5 Cytokines levels in ten mice immunized with Salmonella Choleraesuis vaccines. IFN- $y(\mathbf{A})$, IL-4 (B) or IL-17A (C) in sera at 0.5, 3, and 5 days, in the spleen at 7 days after the boost were assayed with ELISA kit. BSG control was also included. ${ }^{*} P<0.05$; ${ }^{*} P<0.01$, for the cytokines levels induced by strains rSC0011, rSC0016 and rSC0018 containing pS-SaoA compared with that containing the empty vector pYA3493; ${ }^{\#} P<0.05$, ${ }^{\#} P<0.01$ for the significant differences between groups were indicated. The assay was performed in triplicate. The data were collected from two experiments and analyzed. 
Table 2 Vaccine strains carrying plasmid pS-SaoA confer protection against i.p. challenge with SS2 in BALB/c mice

\begin{tabular}{|c|c|c|}
\hline Groups & Genotypes & Percentage of survival (Number of mice Survival/Total) \\
\hline rSC0018(pS-SaoA) & $\triangle a s d A 33$ & $0 \%(0 / 10)$ \\
\hline rSC0018(pYA3493) & $\triangle a s d A 33$ & $0 \%(0 / 10)$ \\
\hline rSC0016(pS-SaoA) & 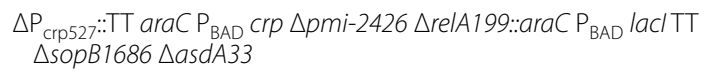 & $100 \% * *(10 / 10)$ \\
\hline rSC0016(pYA3493) & 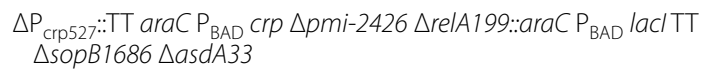 & $0 \%(0 / 10)$ \\
\hline rSC0011(pS-SaoA) & 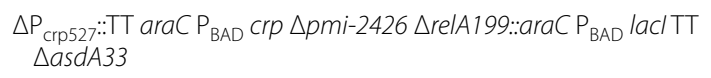 & $40 \%(4 / 10)$ \\
\hline rSC0011(pYA3493) & 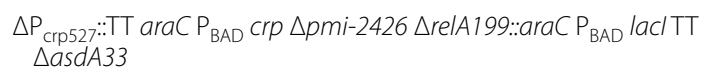 & $0 \%(0 / 10)$ \\
\hline BSG & NA & $0 \%(0 / 10)$ \\
\hline
\end{tabular}

** $P<0.01$ for the survival of mice immunized with rSC0016(pS-SaoA) compared with survival of mice immunized with rSC0011(pS-SaoA) or BSG.

${ }^{a}$ Mice were challenged by i.p. with $25 \times \mathrm{LD}_{50}$ of SS2 at 7 weeks after immunization; This experiment was performed twice with same results. The data showed the result of the single experiment.

with $\mathrm{rSC} 0018(\mathrm{pS}-\mathrm{SaoA})$ were equal to those immunized with rSC0018(pYA3493) at 3 and 5 days after the booster. Significantly higher levels of IFN- $\gamma$, IL-4, and IL-17A were detected in the piglets immunized with rSC0016 carrying either pS-SaoA or pYA3493 compared to those with rSC0018 (Figures 7A-C; $\left.{ }^{\#} P<0.05,{ }^{\# \#} P<0.01\right)$, except in the serum at $12 \mathrm{~h}$ after the booster. Furthermore, the levels of IFN- $\gamma$ and IL-17A in the swine immunized with rSC0016(pSSaoA) were 50 -fold higher in the sera 5 days after the booster compared to those with rSC0018(pS-SaoA) (Figures 7A and D). IL-17, mainly produced by Th17 cells [53-55], can synergize with IFN- $\gamma$ to enhance the production of proinflammatory cytokines [56]. Our results indicated that the new live attenuated strain rSC0016 is more immunogenic than the chemically attenuated strain rSC0018.

\section{Salmonella Choleraesuis vaccine vector with sopB mutation confers protection against SS2 in piglets}

Previous studies showed that the percentage of protection conferred by a specific vaccine in pigs is usually lower than that in mice $[7,10,23]$. Therefore, the protective efficacy of the live attenuated strain rSC0016(pS-SaoA) against SS2 was evaluated in piglets. It provided 100\% protection against 25 times the $\mathrm{LD}_{50}$ of SS2 challenge in mice. Following challenge with $5 \times 10^{8} \mathrm{CFU}$ of SS2 $\left(8.9 \times \mathrm{LD}_{50}\right.$ in piglets $)$ via the ear vein, all the pigs immunized with either rSC0018(pYA3493) or BSG died within $48 \mathrm{~h}$ of challenge (Figure $8 \mathrm{~A}$ ). The piglets immunized with rSC0016(pYA3493) survived longer but died at $84 \mathrm{~h}$ after challenge (Figure 8A). The swine immunized with rSC0018(pS-SaoA) died $108 \mathrm{~h}$ after challenge and showed symptoms of breathing difficulty and joint swelling
(Figures 8A and B). In contrast, all the swine immunized with strain rSC0016(pS-SaoA) survived throughout a 14-day observation period and appeared healthy throughout this period, except for temporary depression within $48 \mathrm{~h}$ of a challenge. A histopathological analysis of the challenged swine immunized with either strain rSC0018(pS-SaoA) or BSG showed hemorrhage, congestion, and inflammatory exudation in the brain (Figure $8 \mathrm{~B}$ ). The swine immunized with strain $\mathrm{rSC} 0016(\mathrm{pS}-\mathrm{SaoA})$ showed normal structures (Figure $8 \mathrm{~B}$ ). These results suggest that strain rSC0016(pS-SaoA) provided excellent protection against $\mathrm{SS} 2$ in the piglet model.

\section{Salmonella Choleraesuis vaccine vector with sopB mutation confers cross-protection against SS7 in piglets}

Sao is a highly conserved protein in S. suis [16]. The protection against SS7, one of the main S. suis serotypes in China, conferred by rSC0016(pS-SaoA) was evaluated in piglets. SS7 is not lethal in pigs and only led to a fever in pigs after intravenous challenge. The cross-reactivity of the sera from pigs immunized with Salmonella Choleraesuis vector rSC0016, carrying SS2 SaoA protein, against SS7 SaoA protein was tested by Western blot. The results showed that the sera from the pigs vaccinated with rSC0016 or rSC0018, carrying SS2 SaoA protein, could recognize the SS7 SaoA protein as well as the SS2 SaoA protein (Figure $8 \mathrm{C}$ ).

At days 1-4 after challenge with SS7, the swine immunized with rSC0016(pYA3493), rSC0018(pYA3493), rSC0018(pS-SaoA), or BSG showed severe fever (Figure $8 \mathrm{D}$ ), drowsiness, and asthma. All these symptoms disappeared 6 days after challenge. In contrast, the swine immunized with rSC0016(pS-SaoA) showed no symptoms during the 7-day observation period. 
- rSC0018(pS-SaoA) rSC0016(pYA3493)

口 rSC0016(pS-SaoA) $\bigcirc$ rSC0018(pYA3493)

A

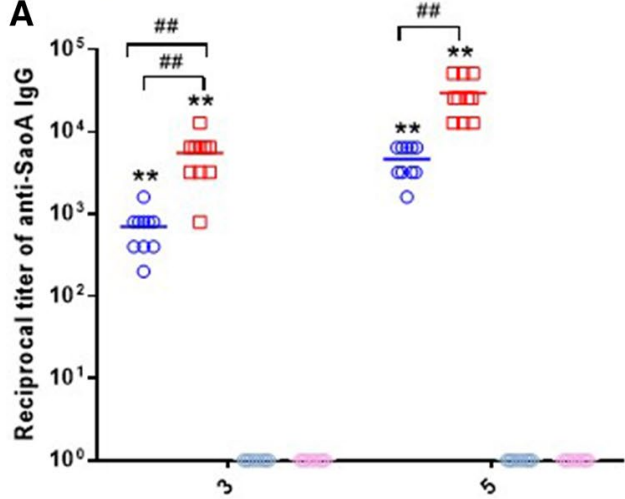

B
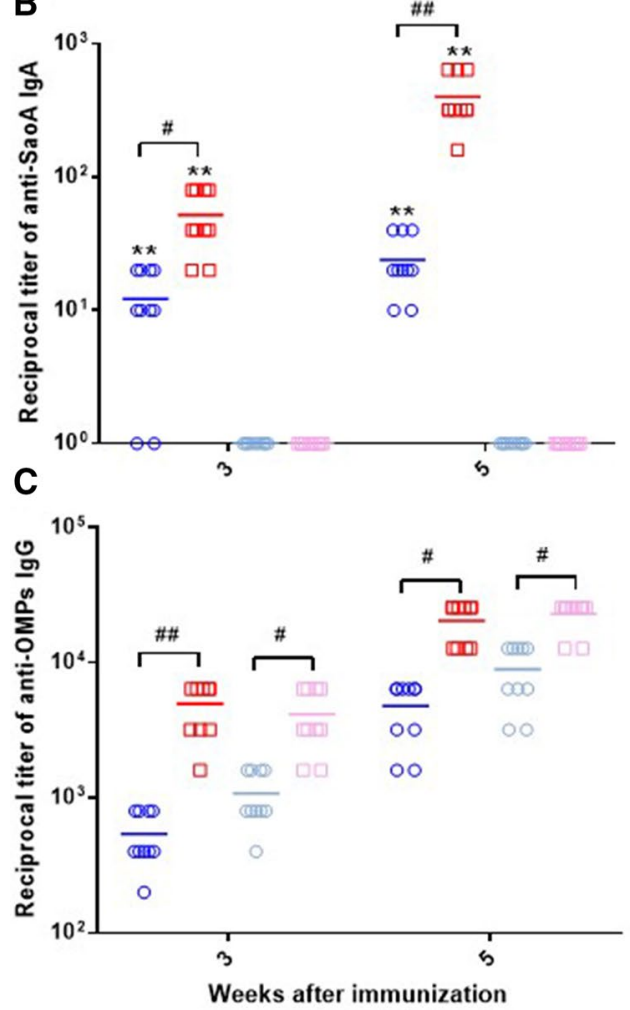

Figure 6 Serum IgG responses to SaoA $(A)$, nasal IgA responses to SaoA (B), serum IgG responses to Salmonella Choleraesuis OMPs $(\mathbf{C})$ in ten pigs were measured by ELISA. The data represent reciprocal anti-lgG antibody titers from the piglets orally immunized with attenuated Salmonella carrying either pS-SaoA and pYA3493 at the indicated weeks after immunization. Serum and nasal cavity wash obtained from individual pig were serially diluted to obtain titers, starting from either 1:50 or 1:10. Error bars represent variation between different pigs. Significant differences were indicated. ${ }^{* *} P<0.01$, for Salmonella Choleraesuis with pS-SaoA compare to Salmonella Choleraesuis with pYA3493 in $\mathbf{A}$ and $\mathbf{B} ;{ }^{\#} P<0.05$; $\# P<0.01$, for Salmonella carrying either pS-SaoA or pYA3493 compared each other. No responses were detected to antigen tested in pigs immunized with BSG or in pre-immune sera from vaccinated piglets. ELISA was performed twice with identical results.

\section{Discussion}

Recently, an innovative Salmonella Choleraesuis live attenuated vaccine vector $\mathrm{rSC} 0011$ with the mutations for regulated delayed attenuation $(\Delta p m i-2426$ deletion mutation, $\Delta \mathrm{P}_{\text {crp527 }}:$ TT $\operatorname{araC} \mathrm{P}_{\mathrm{BAD}}$ crp deletion-insertion mutation) $[29,33]$, and regulated delayed antigen synthesis ( $\triangle$ relA::araC $\mathrm{P}_{\mathrm{BAD}}$ lacI TT deletion-insertion mutation) was developed $[29,57]$. This vaccine vector, delivering a conserved protein of $S$. suis, only conferred protection against a low-dose challenge of SS2 in mice [34], but not a high-dose challenge of SS2 (Table 2). Therefore, it was necessary to improve the immunogenicity of the strain, rSC0011. The SopB protein of Salmonella Typhimurium plays an immunosuppressive role [48], and its inactivation improves humoral and cellular immune responses of the host $[35,48]$. In this study, a $\operatorname{sop} B$ deletion mutation was introduced into our Salmonella Choleraesuis vector with the regulated delayed attenuation system to generate the new strain rSC0016. This new strain displayed the expected phenotypes of reduced fluid secretion and inflammation in rabbit ileal loop test, indicating that the $\triangle \operatorname{sop} B$ deletion in Salmonella Choleraesuis had the same effect as it had in other Salmonella serotypes [52, 58]. Strain rSC0016 showed a similar distribution to that of strain rSC0011 in Peyer's patches of mice, confirming that the sopB mutation did not affect the gut colonization of the strain $[48,52]$. However, the bacterial load of rSC0016 in the spleen was significantly lower than that of rSC0011. This result indicates that strain rSC0016 is more attenuated than rSC0011 in deep lymphoid tissue.

Our results demonstrated that strain rSC0016, with the $\triangle \operatorname{sop} B$ mutation, induces higher levels of IgA, IgG, IFN- $\gamma$, IL- $4 \gamma$ and IL-17A in mice than the other strains tested. These results may relate to the immunosuppressive function of $\operatorname{sop} B$. In addition, it might also be related to the higher antigen loads of rSC0016 in the tissues than those of rSC0018. When Salmonella enters the cell, $10-20 \%$ of the bacteria remain in the cytosol, whereas the majority of the internalized bacteria remain within a modified phagosome, the Salmonella-containing vacuole $[59,60]$. The cytosolic bacteria replicate faster than the vacuolar bacteria and are invasion-primed and-competent [60]. A small but significant amount of the cytosolic bacteria can leave epithelial cells, facilitating a rapid secondary infection and inducing both enteric and systemic infections [60]. The $\operatorname{sop} B$ deletion mutant does not affect the intracellular replication of the bacterium but does induce the early death of these cells, increases lysis, and promotes the transmission of the bacteria [59]. The premature death of epithelial cells exposes the strain to the immune system at a great efficiency, which may contribute to the increased immune responses triggered by rSC0016 compared to rSC0011. 

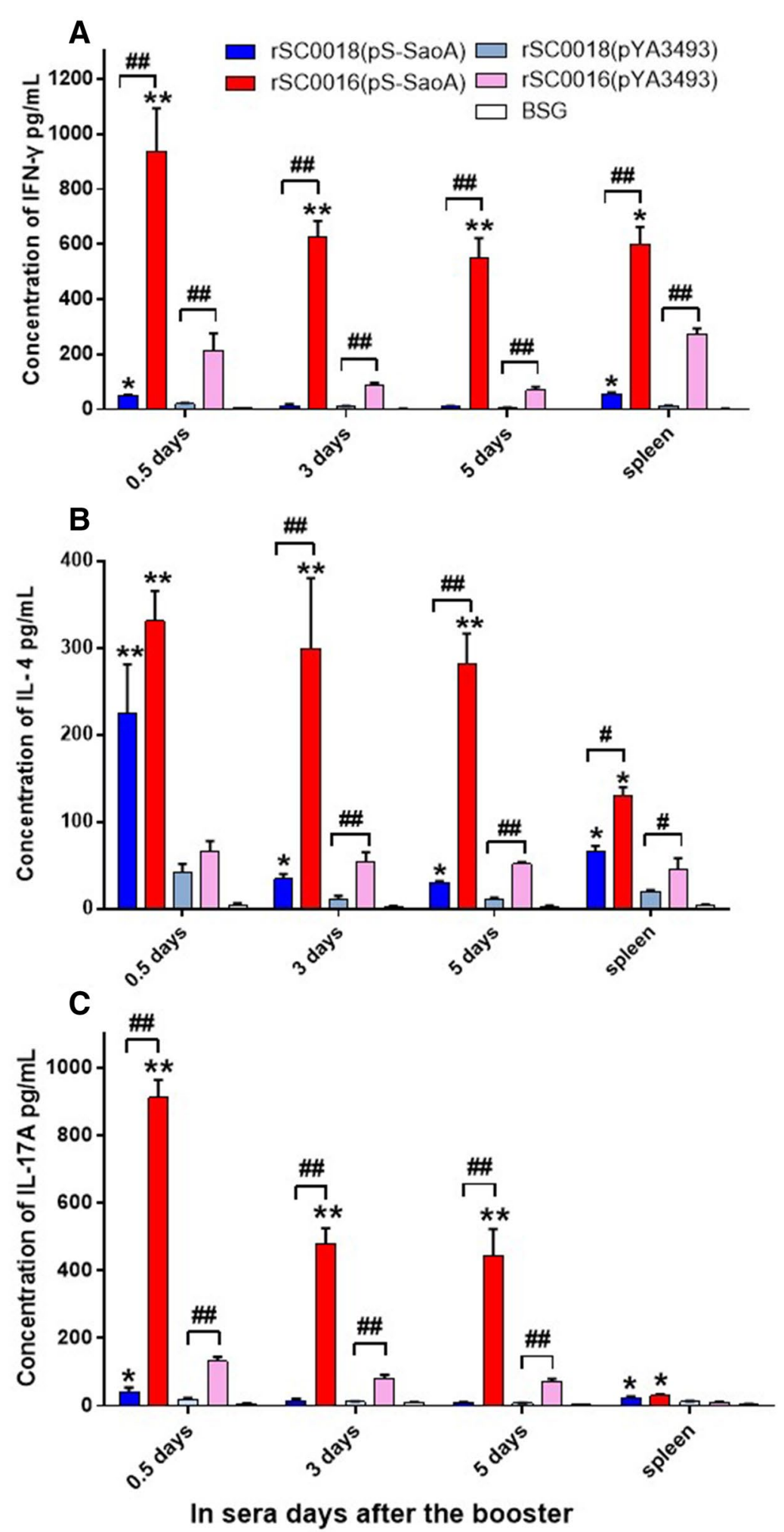

Figure 7 Cytokine levels in ten pigs. IFN- $\boldsymbol{Y}(\mathrm{A})$, IL-4 (B) or IL-17A $(C)$ in sera from $\mathbf{0 . 5}, \mathbf{3}$, and 5 days, in the spleen at 7 days after the booster from single piglet were assayed with ELISA. BSG controls were also included. ${ }^{*} P<0.05 ;{ }^{*} P<0.01$, for the cytokines levels induced by strains rSC0011, rSC0016 and rSC0018 containing PS-SaoA compared with that containing the empty vector pYA3493; $P<0.05$, \#\# $P<0.01$ for significant differences between groups were indicated. The assay was performed in triplicate. The data were collected from two experiments and analyzed. 


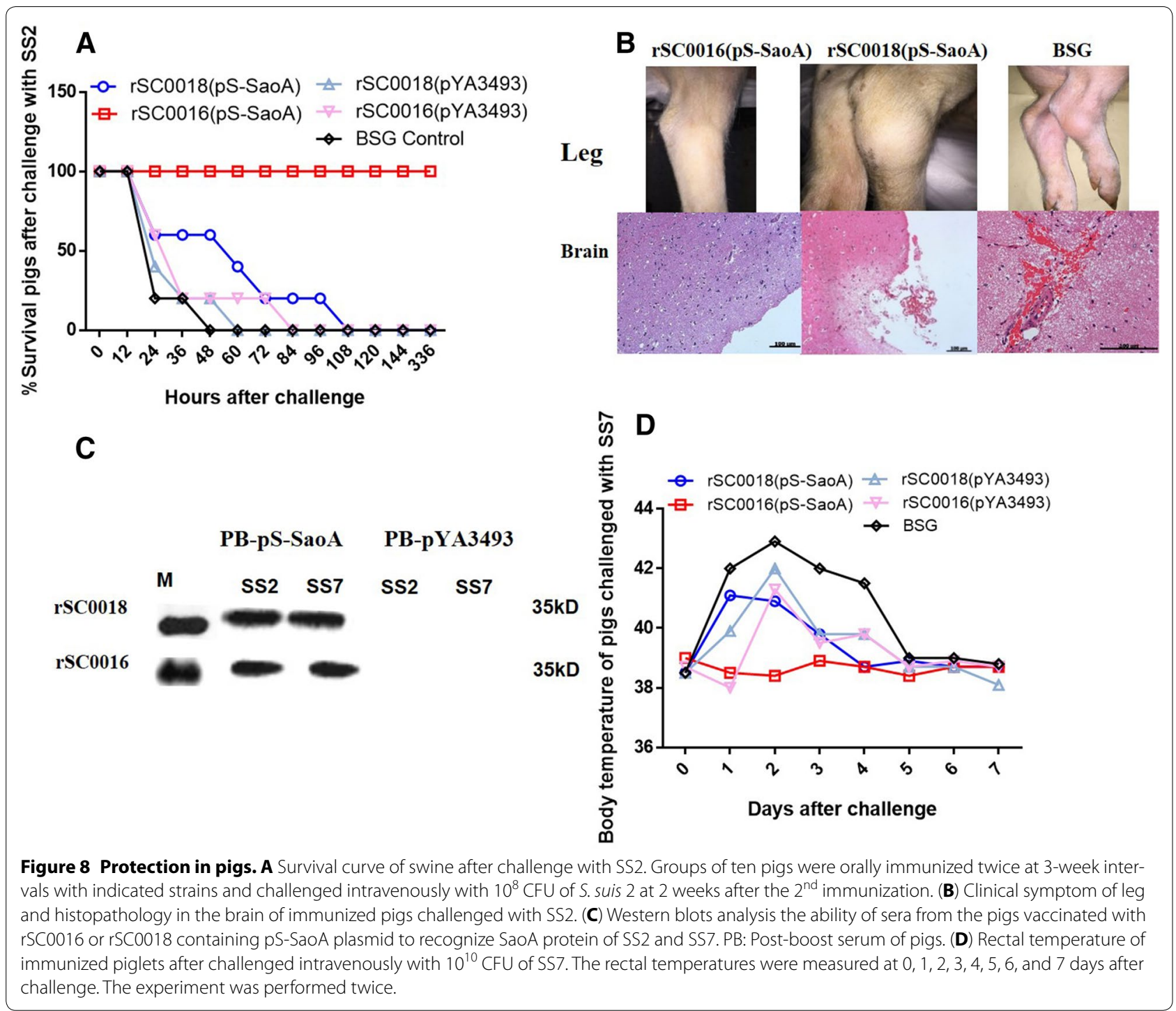

The Salmonella Choleraesuis C500 vaccine induces mucosal IgA and IFN- $\gamma$ responses during its protection of pigs [61], whereas our strain rSC0016 induced greater IgA, IgG, IFN- $\gamma$, IL4, and IL17A responses compared to strain rSC0018, which was derived from C500. IL-17A induces and mediates proinflammatory responses and the expression of many other cytokine genes. The higher levels of IL-17A detected in the sera (34-50 fold) of swine immunized with rSC0016 compared to those with rSC0018 may help the swine clear the infecting pathogen and confer greater protective immunity. The detailed mechanism underlying high IL-17A levels induced by rSC0016 in pig warrants further study.

The immune responses induced by strains rSC0016 and $\mathrm{rSC} 0018$ in both mice and weaned piglets were compared. In mice, the antibody titers of IgG and IgA against Sao of S. suis and IgG against the OMPs of
Salmonella Choleraesuis induced by rSC0016 were significantly higher than those induced by rSC0011. Both were higher than those induced by rSC0018, indicating that the immune responses induced by the combination of the regulated delayed strategy and the $\operatorname{sop} B$ mutation were superior to those induced by the regulated delayed strategy alone or with chemically attenuated rSC0018. The trends in the immune responses of weaned piglets were similar to those of mice. It is noteworthy that the mucosal IgA responses induced by $\mathrm{rSC} 0016$ were significantly higher than those induced by rSC0011 in mice, and were significantly higher than those induced by $\mathrm{rSC} 0018$ in mice or in piglets after immunization. Our orally delivered Salmonella vaccine vectors induced strong mucosal and humoral responses against the Sao antigen (Figures 4 and 6). IgA could prevent the adhesion of pathogens to mucosal cells and improve the protection 
efficiency of vaccine strains. However, current challenge models (i.p. in mice and i.v. in pig) did not address this. Current challenge models focused on the effectiveness of our candidate vaccine against septicemia, since both i.p. and i.v. challenge could induce severe sepsis or even death. If a vaccine candidate could protect the animal from more severe septicemia, it will reduce more severe consequence, even the host is infected with S. suis. Since Sao is not a major virulence factor [62], IgG response is important for the opsonophagocytic killing of S. suis [24]. The role of IgA in the protection against $S$. suis needs to be studied.

Globally, the predominant $S$. suis serotypes isolated from clinical cases of the disease in pigs are serotypes $2,9,3,1 / 2$, and 7 [63]. Preventing the diseases caused by $S$. suis in swine with a universal vaccine is a longsought goal. The Sao protein is highly conserved in $S$. suis [16]. However, cross-protection against serotypes 1 and 7, in addition to SS2, have only been reported in mice $[19,64]$. Whether the Sao protein delivered by Salmonella Choleraesuis could be a universal vaccine for swine against other S. suis serotypes is not known. Our results showed that the sera from the pigs vaccinated with rSC0016 or rSC0018 carrying SS2 SaoA protein recognized the SS7 SaoA protein, which shared 95.2\% amino acid homology with SS2 SaoA (data not show). Piglets immunized with rSC0016(pS-SaoA) had normal body temperatures after challenge with SS7, whereas pigs immunized with either rSC0018(pS-SaoA), a control strain carrying the empty vector, or BSG experienced fever 1-4 days after challenge. This indicates that SaoA delivered by the innovative Salmonella Choleraesuis vector, which combines the regulated delayed strategy and the $\operatorname{sop} B$ mutation, provides cross-protection against SS7.

In conclusion, the new recombinant attenuated Salmonella Choleraesuis vector, which combines the regulated delayed strategy and the $\operatorname{sop} B$ mutation, displays a balance between adequate attenuation and safety, and induces improved immune responses. The new recombinant attenuated Salmonella Choleraesuis strain, synthesizing the SaoA protein, provided full protection against SS2 in mice and pigs. SS7 is not lethal and only leads to fever in pigs by intravenous injection without displaying other clinical signs. Vaccinated pigs did not develop fever after challenge with SS7, indicating that our vaccine is cross-protective against SS7 in swine. In the future, the protection afforded by the new recombinant attenuated Salmonella Choleraesuis vector against other serotypes of S. suis, such as serotypes 9,3 , and $1 / 2$ will be evaluated in pigs. The data reported in this study laid the foundation for the development of a universal vaccine against $S$. suis.
Competing interests

The authors declare that they have no competing interests.

\section{Authors' contributions}

YAL, ZYJ, XBW, SFW and HYS: conceived and designed the experiments. YAL, $Z Y J, X B W$ : performed the experiments. YAL, SFW, and HYS: wrote the paper. All authors read and approved the final manuscript.

\section{Acknowledgements}

This work was supported by the National Natural Science Foundation of China (31672516, 31172300), supported by the Grant No. BE2016343 from Jiangsu province, and the Priority Academic Program Development of Jiangsu Higher Education Institutions (PAPD). We thank Dr Curtiss for kindly providing X7213, pRE112, pYA3493, and the antibody of Lacl. We also thank Matthew Bellefleur for help in editorial advice.

\section{Ethics approval and consent to participate}

Veterinarians took the samples for analysis purposes and/or to check the health status of the mice and pigs population. Both actions do not require ethical approval or were permitted for animal experimentation according to the Jiangsu Administrative Committee for Laboratory Animals (permission number SYXK-SU-2007-0005) and complied with the Jiangsu Laboratory Animal Welfare and Ethics guidelines of the Jiangsu Administrative Committee of Laboratory Animals.

\section{Author details}

${ }^{1}$ College of Veterinary Medicine, Yangzhou University, Yangzhou 225009, Jiangsu, China. ${ }^{2}$ Jiangsu Co-innovation Center for the Prevention and Control of Important Animal Infectious Diseases and Zoonoses, Yangzhou 225009, China. ${ }^{3}$ Department of Infectious Diseases and Pathology, College of Veterinary Medicine, University of Florida, Gainesville, FL 32611-0880, USA.

\section{Publisher's Note}

Springer Nature remains neutral with regard to jurisdictional claims in published maps and institutional affiliations.

Received: 26 May 2017 Accepted: 28 November 2017

Published online: 21 December 2017

\section{References}

1. Goyette-Desjardins G, Auger JP, Xu J, Segura M, Gottschalk M (2014) Streptococcus suis, an important pig pathogen and emerging zoonotic agent-an update on the worldwide distribution based on serotyping and sequence typing. Emerg Microbes Infect 3:e45

2. Kerdsin A, Hatrongjit R, Gottschalk M, Takeuchi D, Hamada S, Akeda Y, Oishi K (2017) Emergence of Streptococcus suis serotype 9 infection in humans. J Microbiol Immunol Infect 50:545-546

3. Segura M, Zheng H, de Greeff A, Gao GF, Grenier D, Jiang Y, Lu C, Maskell D, Oishi K, Okura M, Osawa R, Schultsz C, Schwerk C, Sekizaki T, Smith H, Srimanote P, Takamatsu D, Tang J, Tenenbaum T, Tharavichitkul P, Hoa NT, Valentin-Weigand P, Wells JM, Wertheim H, Zhu B, Xu J, Gottschalk M (2014) Latest developments on Streptococcus suis: an emerging zoonotic pathogen: part 2. Future Microbiol 9:587-591

4. Segura M, Zheng H, de Greeff A, Gao GF, Grenier D, Jiang Y, Lu C, Maskell D, Oishi K, Okura M, Osawa R, Schultsz C, Schwerk C, Sekizaki T, Smith H, Srimanote P, Takamatsu D, Tang J, Tenenbaum T, Tharavichitkul P, Hoa NT, Valentin-Weigand P, Wells JM, Wertheim H, Zhu B, Gottschalk M, Xu J (2014) Latest developments on Streptococcus suis: an emerging zoonotic pathogen: part 1. Future Microbiol 9:441-444

5. Wisselink HJ, Vecht U, Stockhofe-Zurwieden N, Smith HE (2001) Protection of pigs against challenge with virulent Streptococcus suis serotype 2 strains by a muramidase-released protein and extracellular factor vaccine. Vet Rec 148:473-477

6. Quessy S, Dubreuil JD, Caya M, Letourneau R, Higgins R (1994) Comparison of pig, rabbit and mouse IgG response to Streptococcus suis serotype 2 proteins and active immunization of mice against the infection. Can $J$ Vet Res 58:220-223 
7. Zhang A, Chen B, Li R, Mu X, Han L, Zhou H, Chen H, Meilin J (2009) Identification of a surface protective antigen, HP0197 of Streptococcus suis serotype 2. Vaccine 27:5209-5213

8. Li W, Hu X, Liu L, Chen H, Zhou R (2011) Induction of protective immune response against Streptococcus suis serotype 2 infection by the surface antigen HP0245. FEMS Microbiol Lett 316:115-122

9. Okwumabua O, Chinnapapakkagari S (2005) Identification of the gene encoding a 38-kilodalton immunogenic and protective antigen of Streptococcus suis. Clin Diagn Lab Immunol 12:484-490

10. Tan C, Liu M, Liu J, Yuan F, Fu S, Liu Y, Jin M, Bei W, Chen H (2009) Vaccination with Streptococcus suis serotype 2 recombinant 6PGD protein provides protection against S. suis infection in swine. FEMS Microbiol Lett 296:78-83

11. Chen B, Zhang A, Li R, Mu X, He H, Chen H, Jin M (2010) Evaluation of the protective efficacy of a newly identified immunogenic protein, $\mathrm{HP} 0272$, of Streptococcus suis. FEMS Microbiol Lett 307:12-18

12. Seele J, Hillermann LM, Beineke A, Seitz M, von Pawel-Rammingen $U$, Valentin-Weigand P, Baums CG (2015) The immunoglobulin M-degrading enzyme of Streptococcus suis, Ide Ssuis, is a highly protective antigen against serotype 2. Vaccine 33:2207-2212

13. Tan C, Fu S, Liu M, Jin M, Liu J, Bei W, Chen H (2008) Cloning, expression and characterization of a cell wall surface protein, 6-phosphogluconate-dehydrogenase, of Streptococcus suis serotype 2. Vet Microbiol 130:363-370

14. Jacobs AA, van den Berg AJ, Loeffen PL (1996) Protection of experimentally infected pigs by suilysin, the thiol-activated haemolysin of Streptococcus suis. Vet Rec 139:225-228

15. Liu L, Cheng G, Wang C, Pan X, Cong Y, Pan Q, Wang J, Zheng F, Hu F, Tang $J$ (2009) Identification and experimental verification of protective antigens against Streptococcus suis serotype 2 based on genome sequence analysis. Curr Microbiol 58:11-17

16. LiY, Martinez G, Gottschalk M, Lacouture S, Willson P, Dubreuil JD, Jacques M, Harel J (2006) Identification of a surface protein of Streptococcus suis and evaluation of its immunogenic and protective capacity in pigs. Infect Immun 74:305-312

17. Garibaldi M, Rodriguez-Ortega MJ, Mandanici F, Cardaci A, Midiri A, Papasergi S, Gambadoro O, Cavallari V, Teti G, Beninati C (2010) Immunoprotective activities of a Streptococcus suis pilus subunit in murine models of infection. Vaccine 28:3609-3616

18. Mandanici F, Gomez-Gascon L, Garibaldi M, Olaya-Abril A, Luque I, Tarradas C, Mancuso G, Papasergi S, Barcena JA, Teti G, Beninati C, RodriguezOrtega MJ (2010) A surface protein of Streptococcus suis serotype 2 identified by proteomics protects mice against infection. J Proteom 73:2365-2369

19. Hsueh KJ, Lee JW, Hou SM, Chen HS, Chang TC, Chu CY (2014) Evaluation on a Streptococcus suis vaccine using recombinant sao-I protein manufactured by bioreactors as the antigen in pigs. Transbound Emerg Dis 61:e35-e43

20. Feng Y, Pan X, Sun W, Wang C, Zhang H, Li X, Ma Y, Shao Z, Ge J, Zheng F, Gao GF, Tang J (2009) Streptococcus suis enolase functions as a protective antigen displayed on the bacterial cell surface. J Infect Dis 200:1583-1592

21. Gomez-Gascon L, Cardoso-Toset F, Amarilla PS, Tarradas C, Carrasco L, Olaya-Abril A, Jimenez-Munguia I, Rodriguez-Ortega MJ, Luque I (2014) A new recombinant SnA protein combined with aluminum hydroxide protects mouse against Streptococcus suis. Vaccine 32:6992-6999

22. Huang K, Yuan Z, Li J, Zhang Q, Xu Z, Yan S, Zhang A, Jin M (2015) Identification and characterisation a surface-associated arginine peptidase in Streptococcus suis serotype 2. Microbiol Res 170:168-176

23. Li J, Xia J, Tan C, Zhou Y, Wang Y, Zheng C, Chen H, Bei W (2011) Evaluation of the immunogenicity and the protective efficacy of a novel identified immunogenic protein, SsPepO, of Streptococcus suis serotype 2. Vaccine 29:6514-6519

24. Li Y, Gottschalk M, Esgleas M, Lacouture S, Dubreuil JD, Willson P, Harel J (2007) Immunization with recombinant Sao protein confers protection against Streptococcus suis infection. Clin Vaccine Immunol 14:937-943

25. Chabot-Roy G, Willson P, Segura M, Lacouture S, Gottschalk M (2006) Phagocytosis and killing of Streptococcus suis by porcine neutrophils. Microb Pathog 41:21-32

26. Park SM, Ko HJ, Shim DH, Yang JY, Park YH, Curtiss R $3^{\text {rd }}$, Kweon MN (2008) MyD88 signaling is not essential for induction of antigen-specific $B$ cell responses but is indispensable for protection against Streptococcus pneumoniae infection following oral vaccination with attenuated Salmonella expressing PspA antigen. J Immunol 181:6447-6455

27. Curtiss R $3^{\text {rd }}$ (2002) Bacterial infectious disease control by vaccine development. J Clin Invest 110:1061-1066

28. Galen JE, Levine MM (2001) Can a'flawless'live vector vaccine strain be engineered? Trends Microbiol 9:372-376

29. Curtiss $R 3^{\text {rd }}$, Wanda S-Y, Gunn BM, Zhang $X$, Tinge SA, Ananthnarayan V, Mo H, Wang S, Kong W (2009) Salmonella enterica serovar Typhimurium strains with regulated delayed attenuation in vivo. Infect Immun 77:1071-1082

30. Wang S, Li Y, Scarpellini G, Kong W, Shi H, Baek CH, Gunn B, Wanda SY, Roland KL, Zhang X, Senechal-Willis P, Curtiss R $3^{\text {rd }}$ (2010) Salmonella vaccine vectors displaying delayed antigen synthesis in vivo to enhance immunogenicity. Infect Immun 78:3969-3980

31. Collins LV, Attridge S, Hackett J (1991) Mutations at rfc or pmi attenuate Salmonella typhimurium virulence for mice. Infect Immun 59:1079-1085

32. Katzman RL, Lisowska E, Jeanloz RW (1970) Invertebrate connective tissue. Isolation of D-arabinose from sponge acidic polysaccharide. Biochem J 119:17-19

33. Shi H, Santander J, Brenneman KE, Wanda SY, Wang S, Senechal P, Sun W, Roland KL, Curtiss R $3^{\text {rd }}$ (2010) Live recombinant Salmonella Typhi vaccines constructed to investigate the role of rpos in eliciting immunity to a heterologous antigen. PLoS One 5:e11142

34. Ji Z, Shang J, Li Y, Wang S, Shi H (2015) Live attenuated Salmonella enterica serovar Choleraesuis vaccine vector displaying regulated delayed attenuation and regulated delayed antigen synthesis to confer protection against Streptococcus suis in mice. Vaccine 33:4858-4867

35. Li Y, Wang S, Xin W, Scarpellini G, Shi Z, Gunn B, Roland KL, Curtiss R $3^{\text {rd }}$ (2008) A sopB deletion mutation enhances the immunogenicity and protective efficacy of a heterologous antigen delivered by live attenuated Salmonella enterica vaccines. Infect Immun 76:5238-5246

36. Zhao Z, Xue Y, Wu B, Tang X, Hu R, Xu Y, Guo A, Chen H (2008) Subcutaneous vaccination with attenuated Salmonella enterica serovar Choleraesuis C500 expressing recombinant filamentous hemagglutinin and pertactin antigens protects mice against fatal infections with both S. enterica serovar Choleraesuis and Bordetella bronchiseptica. Infection Immun 76:2157-2163

37. Li Q, Hu Y, Xu L, Xie X, Tao M, Jiao X (2014) Complete genome sequence of Salmonella enterica serovar Choleraesuis vaccine strain C500 attenuated by chemical mutation. Genome Announc 2:e01022-e01024

38. Bertani G (1951) Studies on lysogenesis. I. The mode of phage liberation by lysogenic Escherichia coli. J Bacteriol 62:293-300

39. Curtiss R $3^{\text {rd }}$ (1965) Chromosomal aberrations associated with mutations to bacteriophage resistance in Escherichia Coli. J Bacteriol 89:28-40

40. Briles DE, King JD, Gray MA, McDaniel LS, Swiatlo E, Benton KA (1996) PspA, a protection-eliciting pneumococcal protein: immunogenicity of isolated native PspA in mice. Vaccine 14:858-867

41. Gunn BM, Wanda SY, Burshell D, Wang C, Curtiss R $3^{\text {rd }}$ (2010) Construction of recombinant attenuated Salmonella enterica serovar Typhimurium vaccine vector strains for safety in newborn and infant mice. Clin Vaccine Immunol 17:354-362

42. Shi I (2009) Study on triple genetic engineering vaccine of Salmonella enterica serovar Choleraesuis swine Streptococcus. Master Thesis, Hua Zhong Agriculture University, China

43. Schindelin J, Rueden CT, Hiner MC, Eliceiri KW (2015) The ImageJ ecosystem: an open platform for biomedical image analysis. Mol Reprod Dev 82:518-529

44. Xin W, Wanda S-Y, Li Y, Wang S, Mo H, Curtiss R $3^{\text {rd }}$ (2008) Analysis of type II secretion of recombinant pneumococcal PspA and PspC in a Salmonella enterica Serovar Typhimurium vaccine with regulated delayed antigen synthesis. Infect Immun 76:3241-3254

45. Fusco WG, Afonina G, Nepluev I, Cholon DM, Choudhary N, Routh PA, Almond GW, Orndorff PE, Staats H, Hobbs MM, Leduc I, Elkins C (2010) Immunization with the Haemophilus ducreyi hemoglobin receptor HgbA with adjuvant monophosphoryl lipid A protects swine from a homologous but not a heterologous challenge. Infect Immun 78:3763-3772

46. Kang HY, Srinivasan J, Curtiss R $3^{\text {rd }}$ (2002) Immune responses to recombinant pneumococcal PspA antigen delivered by live attenuated Salmonella enterica serovar typhimurium vaccine. Infect Immun 70:1739-1749

47. Klaasen HLBM, Segers RPAM (2012) Vaccine for protection against Streptococcus suis bacteria of various serotypes. Patent EP2411047A1, 1 Feb 2012 
48. Link C, Ebensen T, Standner L, Dejosez M, Reinhard E, Rharbaoui F, Guzman CA (2006) An SopB-mediated immune escape mechanism of Salmonella enterica can be subverted to optimize the performance of live attenuated vaccine carrier strains. Microbes Infect 8:2262-2269

49. Galyov EE, Wood MW, Rosqvist R, Mullan PB, Watson PR, Hedges S, Wallis TS (1997) A secreted effector protein of Salmonella dublin is translocated into eukaryotic cells and mediates inflammation and fluid secretion in infected ileal mucosa. Mol Microbiol 25:903-912

50. Nakayama KKSM, Curtiss R $3^{\text {rd }}$ (1988) Construction of an ASD + expression-cloning vector: stable maintenance and high level expression of cloned genes in a Salmonella vaccine strain. Nat Biotech 6:693-697

51. Zhang S, Santos RL, Tsolis RM, Stender S, Hardt WD, Baumler AJ, Adams LG (2002) The Salmonella enterica serotype typhimurium effector proteins SipA, SopA, SopB, SopD, and SopE2 act in concert to induce diarrhea in calves. Infect Immun 70:3843-3855

52. Reis BP, Zhang S, Tsolis RM, Baumler AJ, Adams LG, Santos RL (2003) The attenuated SOpB mutant of Salmonella enterica serovar Typhimurium has the same tissue distribution and host chemokine response as the wild type in bovine Peyer's patches. Vet Microbiol 97:269-277

53. Aarvak TCM, Miossec P, Natvig JB (1999) IL-17 is produced by some proinflammatory Th1/Th0 cells but not by Th2 cells. J Immunol 162:1246-1251

54. Sassu EL, Ladinig A, Talker SC, Stadler M, Knecht C, Stein H, Frombling J, Richter B, Spergser J, Ehling-Schulz M, Graage R, Hennig-Pauka I, Gerner W (2017) Frequency of Th17 cells correlates with the presence of lung lesions in pigs chronically infected with Actinobacillus pleuropneumoniae. Vet Res 48:4

55. Stepanova H, Mensikova M, Chlebova K, Faldyna M (2012) CD4 + and gdTCR + T lymphocytes are sources of interleukin-17 in swine. Cytokine 58:152-157

56. Teunissen MB, Koomen CW, de Waal Malefyt R, Wierenga EA, Bos JD (1998) Interleukin-17 and interferon-gamma synergize in the enhancement of proinflammatory cytokine production by human keratinocytes. J Invest Dermatol 111:645-649
57. Shi H, Wang S, Curtiss R $3^{\text {rd }}$ (2013) Evaluation of regulated delayed attenuation strategies for Salmonella enterica serovar Typhi vaccine vectors in neonatal and infant mice. Clin Vaccine Immunol 20:931-944

58. Norris FA, Wilson MP, Wallis TS, Galyov EE, Majerus PW (1998) SopB, a protein required for virulence of Salmonella dublin, is an inositol phosphate phosphatase. Proc Natl Acad Sci U S A 95:14057-14059

59. Finn CE, Chong A, Cooper KG, Starr T, Steele-Mortimer O (2017) A second wave of Salmonella T3SS1 activity prolongs the lifespan of infected epithelial cells. PLoS Pathog 13:e1006354

60. Knodler LA, Vallance BA, Celli J, Winfree S, Hansen B, Montero M, Steele-Mortimer O (2010) Dissemination of invasive Salmonella via bacterial-induced extrusion of mucosal epithelia. Proc Natl Acad Sci U S A 107:17733-17738

61. Zhu L, Zhao X, Yin Q, Liu X, Chen X, Huang C, Suo X (2017) Mucosal IgA and IFN-g ${ }^{+}$CD8 $T$ cell immunity are important in the efficacy of live Salmonella enteria serovar Choleraesuis vaccines. Sci Rep 7:46408

62. Roy D, Fittipaldi N, Dumesnil A, Lacouture S, Gottschalk M (2014) The protective protein Sao (surface antigen one) is not a critical virulence factor for Streptococcus suis serotype 2. Microb Pathog 67-68:31-35

63. Segura M (2015) Streptococcus suis vaccines: candidate antigens and progress. Expert Rev Vaccines 14:1587-1608

64. Zhang A, Chen B, Mu X, Li R, Zheng P, Zhao Y, Chen H, Jin M (2009) Identification and characterization of a novel protective antigen, Enolase of Streptococcus suis serotype 2. Vaccine 27:1348-1353

65. Kang K (2008) Chinese veterinary strains directory. China agricultural science and technology press, Beijing

\section{Submit your next manuscript to BioMed Central and we will help you at every step:}

- We accept pre-submission inquiries

- Our selector tool helps you to find the most relevant journal

- We provide round the clock customer support

- Convenient online submission

- Thorough peer review

- Inclusion in PubMed and all major indexing services

- Maximum visibility for your research

Submit your manuscript at www.biomedcentral.com/submit
O Biomed Central 